\title{
Synthesis and X-ray diffraction structures of novel half-sandwich Os(II)-and $\mathrm{Ru}(\mathrm{II})$-hydroxamate complexes $\dagger$
}

\author{
Attila J. Godó, ${ }^{a}$ Attila Cs. Bényei, ${ }^{b}$ Brian Duff, ${ }^{c d}$ Denise A. Egan ${ }^{c d}$ and Péter Buglyó*a \\ Received 2nd November 2011, Accepted 7th November 2011 \\ DOI: 10.1039/c1ra00998b
}

Novel water soluble half-sandwich complexes of the general formulae $\left[\mathrm{M}\left(\eta^{6}-p \text {-cym }\right)(\text { ha })\right]_{2}\left(\mathrm{CF}_{3} \mathrm{SO}_{3}\right)_{2}$, $\left[\mathrm{M}\left(\eta^{6}-p\right.\right.$-cym $\left.)(\mathrm{ha}) \mathrm{Cl}\right]$ or $\left[\mathrm{M}\left(\eta^{6}-p\right.\right.$-cym $\left.)(\mathrm{ha})(\mathrm{py})\right] X(M=\mathrm{Os}, \mathrm{Ru}$; ha = hydroxamate; py = pyridine; $X=\mathrm{Cl}^{-}$or $\left.\mathrm{CF}_{3} \mathrm{SO}_{3}{ }^{-}\right)$, incorporating metal-containing entities and hydroxamates both with potential anti-proliferative features, were prepared and characterized by elemental analysis, spectroscopy (NMR, IR) and ESI mass spectrometry. The X-ray crystal structure of $\left[\mathrm{Ru}\left(\eta^{6}-p\right.\right.$-cym $)(\mu \text {-meaha) }]_{2}\left(\mathrm{CF}_{3} \mathrm{SO}_{3}\right)_{2}(\mathbf{5}),\left[\mathrm{Os}\left(\eta^{6}-p\right.\right.$-cym $)($ meaha $\left.) \mathrm{Cl}\right](\mathbf{6}),\left[\mathrm{Ru}\left(\eta^{6}-p\right.\right.$-cym $\left.)(\mathrm{phebha}) \mathrm{Cl}\right]$, (9), $\left[\mathrm{Ru}\left(\eta^{6}-p\right.\right.$-cym)(bha)(py)]( $\left.\mathrm{CF}_{3} \mathrm{SO}_{3}\right)(\mathbf{1 2})$ and $\left[\mathrm{Ru}\left(\eta^{6}-p\right.\right.$-cym $)($ phebha $\left.)(\mathrm{py})\right]\left(\mathrm{CF}_{3} \mathrm{SO}_{3}\right)(\mathbf{1 4}), 6$ is the first published structure of an organometallic Os(II)-hydroxamate reported. The effect of size differences of the metal ions, the steric demand of the $R_{C}$ and $R_{N}$ substituents at the hydroxamate group and the type of the monodentate ligand co-present in the stoichiometry, along with the binding architecture of the half-sandwich metal(II) hydroxamate complexes are discussed. A novel dinuclear, dihydroxo bridged complex $\left[\mathrm{Os}\left(\eta^{6}-p \text {-cym }\right)(\mathrm{py})(\mu-\mathrm{OH})\right]_{2}\left(\mathrm{CF}_{3} \mathrm{SO}_{3}\right)_{2}(\mathbf{1 6})$ is prepared and characterized by X-ray crystallography. Unexpected formation of a dinuclear oxo bridged $\mathrm{Os}^{\mathrm{II}} / \mathrm{Os}^{\mathrm{VI}}$ complex $\left[\left\{\mathrm{Os}\left(\eta^{6}-p\right.\right.\right.$-cym $\left.\left.)(\mathrm{meaha})\right\}(\mu-\mathrm{O})\left\{\mathrm{Os}(\mathrm{O})(\text { meaha })_{2}\right\}\right] \mathrm{Cl}(\mathbf{1 7})$ occurs, and the crystal and molecular structure has been determined by X-ray method. Complexes 1, 5-8, 10 and 14 were tested for their in vitro cytotoxicity, using human-derived ovarian cancer cell lines (A2780 and A2780 cisR), and showed no anti-proliferative effect in the concentration range $(0-200 \mu \mathrm{M})$ studied.

\section{Introduction}

Hydroxamic acids, $\mathrm{R}^{1} \mathrm{CON}\left(\mathrm{R}^{2}\right) \mathrm{OH}$, are an important class of biomolecules, capable of forming stable five-membered $(\mathrm{O}, \mathrm{O})$ chelates with a wide range of metal ions. This strong interaction may result in an essential role for these ligands in terms of uptake and transport of different metal ions, e.g. $\mathrm{Fe}^{3+}$, mainly in microorganisms, or in the effective and selective inhibition of various metalloenzymes. ${ }^{1}$ Based on the inhibition of histone deacetylases, a monohydroxamic acid, suberoilanilide hydroxamic acid (sahaH), is currently undergoing clinical use as a treatment for cutaneous T-cell lymphoma. ${ }^{2,3}$

Half-sandwich $\mathrm{Ru}(\mathrm{II})$ complexes with promising anti-proliferative properties have also been the subject of intensive research in recent decades. Among others, the effects of the size and

${ }^{a}$ Department of Inorganic and Analytical Chemistry, University of Debrecen, H-4010 Debrecen, Hungary.E-mail: buglyo@science.unideb.hu ${ }^{b}$ Laboratory for X-ray Diffraction, Institute of Chemistry, Univesity of Debrecen, H-4010 Debrecen, Hungary

${ }^{c}$ Centre for Pharmaceutical Research and Development, Institute of Technology Tallaght, Dublin 24, Ireland.

${ }^{d}$ Department of Science, Institute of Technology Tallaght, Dublin 24, Ireland.

$\uparrow$ Electronic supplementary information (ESI) available. CCDC reference numbers 838351, 838352, 838353, 838354, 838355, 838356 and 838357. For ESI and crystallographic data in CIF or other electronic format see DOI: 10.1039/c1ra00998b hydrophobicity of the $\eta^{6}$-arene, the type of the coordinated $(\mathrm{N}, \mathrm{N})(\mathrm{N}, \mathrm{O})$ or $(\mathrm{O}, \mathrm{O})$ chelating ligands, the rate of aquation of the monodentate ligand at the sixth coordination site, and the acidity of the water molecule after aquation have all been studied on the in vitro cytotoxicity of the complexes. ${ }^{4-7}$

We have hypothesized that a combination of the two entities, namely hydroxamate and $\left[\mathrm{Ru}^{\mathrm{II}}\left(\eta^{6}\right.\right.$-arene)], into one molecule may result in the production of molecules with beneficial properties. ${ }^{8,9}$ Indeed, preliminary results have shown that $\left[\mathrm{Ru}\left(\eta^{6}-p\right.\right.$-cym)(saha) $\mathrm{Cl}$ ] has moderate cytotoxicity $\left(\mathrm{IC}_{50}=85 \mu \mathrm{M}\right)$ against two ovarian cancer cell lines, namely A2780 and A2780 cisR. ${ }^{10}$

However, significantly less is known about the corresponding osmium complexes with the $\left[\mathrm{Os}^{\mathrm{II}}\left(\eta^{6}\right.\right.$-arene $\left.)\right]$ entity. Half-sandwich osmium(II) compounds with $(\mathrm{N}, \mathrm{N})^{11-14}(\mathrm{~N}, \mathrm{O})^{15,16}$ or $(\mathrm{O}, \mathrm{O})^{11,17}$ chelating ligands have been synthesized and tested against different cancer cell lines. In particular, acetylacetonato ${ }^{11}$ or maltolato ${ }^{17}$ containing complexes, $\left[\mathrm{Os}\left(\eta^{6}-p\right.\right.$-cym $\left.)(\mathrm{O}, \mathrm{O}) \mathrm{Cl}\right]$, were found to be capable of fast ligand exchange in aqueous solution, resulting in the formation of an inactive hydroxo species at physiological $\mathrm{pH}$. In the case of hydroxamates as $(\mathrm{O}, \mathrm{O})$ donors interacting with any forms of Os, only one report was found in the literature. Here, a reaction of $\left[\mathrm{Os}^{\mathrm{II}}(\mathrm{bpy})_{2} \mathrm{Br}_{2}\right]$ (bpy $=2,2^{\prime}$-bipyridine) with $\mathrm{N}$-arylbenzohydroxamic acids produced a cyclometalate of Os(III). The benzanilide which was formed from the hydroxamate coordinated as a dianionic $\mathrm{C}, \mathrm{N}$-donor. ${ }^{18}$ To our 
knowledge, there are no reports on Os complexes with $(\mathrm{O}, \mathrm{O})$ coordinated hydroxamate in the literature.

A comparison of the rate of ligand exchange reactions in halfsandwich $\mathrm{Ru}$ and Os complexes, $\left[\mathrm{M}\left(\eta^{6}\right.\right.$-arene $\left.)(\mathrm{XY}) \mathrm{Z}\right]$, indicates that the rate is $3-5$ orders of magnitude smaller in Os complexes than in their corresponding ruthenium analogues. ${ }^{11}$ In general, while $(\mathrm{N}, \mathrm{N})$ donor ligand containing $\mathrm{Ru}$ complexes typically have suitable kinetic inertness and therefore high antiproliferative activity, the corresponding Os complexes might be less active due to the above kinetic differences. On the other hand, while $(\mathrm{O}, \mathrm{O})$ chelated $\mathrm{Ru}$ complexes can be too labile, the Os analogues might be of the appropriate kinetic behaviour and thus exert greater biological activity. Deprotonation of the water molecule after replacing $\mathrm{Z}$ auxiliary ligand in aqueous solution may also result in the formation of an inactive hydroxo species, and this is more pronounced for Os than $\mathrm{Ru}$ complexes with a given $\mathrm{XY}$ chelator. ${ }^{17}$ Since strongly coordinating $\mathrm{XY}$ are capable of shifting this process above $\mathrm{pH} 7.4$, it is possible that Oshydroxamates might yield good potential drug candidates.

Recently we have found that reaction of $\left[\mathrm{Ru}\left(\eta^{6}-p-c y m\right) \mathrm{Cl}_{2}\right]_{2}$ with benzohydroxamate (Chart 1) in the presence of noncoordinating triflate counter ion produced a dinuclear complex in which two half-sandwich metal centers were linked together with two hydroxamates. In this structure the carbonyl oxygens of the bha ligands coordinate to one of the $\mathrm{Ru}$ units, and the hydroxamate oxygens bridge to the two $\mathrm{Ru}$ atoms. ${ }^{8}$ In contrast, spectroscopic and MS results suggest that the presence of other ligands (e.g. $\mathrm{Cl}^{-}$) allows monodentate coordination yielding monomeric $\left[\mathrm{Ru}\left(\eta^{6}-p-c y m\right)(h a) \mathrm{Cl}\right]$ (ha = hydroxamate) type species in solution. ${ }^{8}$
In the current study, we sought to gain deeper insight into the effect of the size of the metal ion ( $\mathrm{Ru} v$ s. Os), the steric demand of the $R_{\mathrm{C}}$ and $R_{\mathrm{N}}$ substituents at the hydroxamate group, the effect of the coordination of primary $\left(R_{\mathrm{N}}=\mathrm{H}\right)$ or secondary $\left(R_{\mathrm{N}}\right.$ $=$ alkyl or aryl) hydroxamate (Chart 1$)$ and the role of any monodentate ligand $\left(\mathrm{Cl}^{-}\right.$, pyridine) may exert on the stability, stoichiometry, nuclearity and binding architecture of the halfsandwich metal(II) hydroxamate complexes. Herein we report the syntheses, solid state characterization and X-ray structures of a series osmium and ruthenium hydroxamate complexes, together with their in vitro anti-cancer potential using human ovarian cancer cell lines (A2780 and A2780cisR).

\section{Experimental}

\subsection{Starting materials}

$\mathrm{OsO}_{4}, \quad \mathrm{RuCl}_{3} \cdot x \mathrm{H}_{2} \mathrm{O}, \quad \alpha$-terpinene, $\mathrm{N}$-methylhydroxylamine hydrochloride, $\mathrm{AgCF}_{3} \mathrm{SO}_{3}$, acetyl chloride, benzoyl chloride, nitrobenzene, pyridine, sodium methoxide, benzohydroxamic acid, $\left[\mathrm{Pt}\left(\mathrm{NH}_{3}\right)_{2} \mathrm{Cl}_{2}\right]$ were commercial products of the highest purity available (Heraeus, Aldrich, Merck or Fluka), and used as received. Solvents were dried and distilled according to standard methods. ${ }^{19} \mathrm{~N}$-phenylhydroxylamine hydrochloride was prepared from nitrobenzene while N-methyl-acetohydroxamic, N-phenylacetohydroxamic and $\mathrm{N}$-phenyl-benzohydroxamic acid were prepared from acetyl or benzoyl chloride, by reaction with $\mathrm{N}$-methyl- or N-phenylhydroxylamine following literature methods. ${ }^{20,21} \mathrm{H}_{2}\left[\mathrm{OsCl}_{6}\right]$ was obtained as an orange-red viscous oil after refluxing $\mathrm{OsO}_{4}$ in an excess of concentrated aqueous $\mathrm{HCl}$ for $40 \mathrm{~h}$, and removing any liquid by rotary evaporation under<smiles>[R]C(=O)N([R3])O</smiles>

$$
\begin{array}{ll}
\mathrm{R}_{\mathrm{N}}=\mathrm{H} & \text { primary } \\
\mathrm{R}_{\mathrm{N}}=\mathrm{R}, \mathrm{Ar} & \text { secondary }
\end{array}
$$<smiles>CN(O)C(=O)c1ccccc1</smiles>

$\mathrm{N}$-methyl-benzohydroxamic acid, mebhaH<smiles>CC(=O)N(C)O</smiles>

$\mathrm{N}$-methyl-acetohydroxamic acid, meahaH<smiles>O=C(c1ccccc1)N(O)c1ccccc1</smiles>

$\mathrm{N}$-phenyl-benzohydroxamic acid, phebhaH<smiles>O=C(NO)c1ccccc1</smiles>

benzohydroxamic acid bhaH<smiles>CC(=O)N(O)c1ccccc1</smiles>

$\mathrm{N}$-phenyl-acetohydroxamic acid, pheahaH

Chart 1 


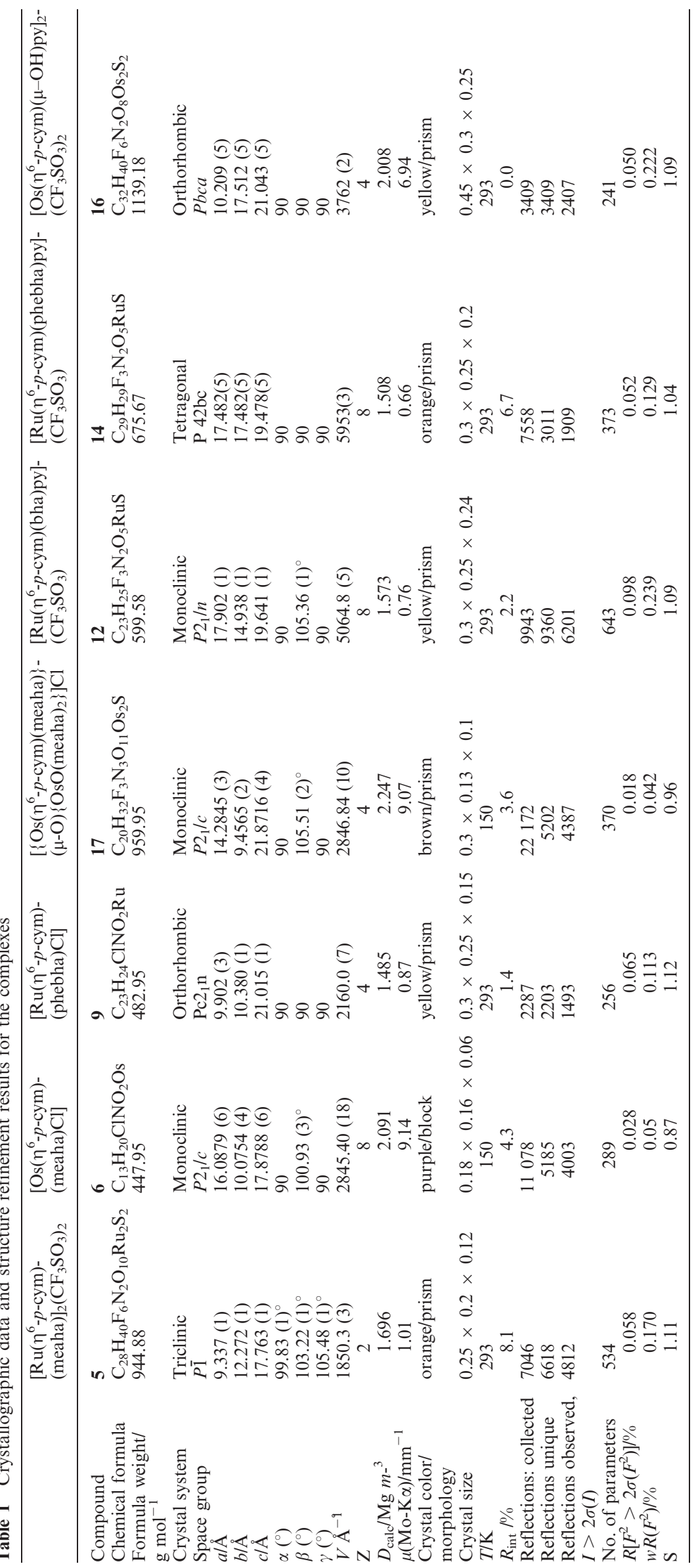


reduced pressure. ${ }^{22}\left[\mathrm{Ru}\left(\eta^{6}-p \text {-cym }\right) \mathrm{Cl}_{2}\right]_{2}$ and $\left[\mathrm{Ru}\left(\eta^{6}-p\right.\right.$-cym $)$ (acetone $\left.)_{3}\right]\left(\mathrm{CF}_{3} \mathrm{SO}_{3}\right)_{2}$ were synthesized and purified according to literature methods. ${ }^{23,24}\left[\mathrm{Os}\left(\eta^{6}-p \text {-cym }\right) \mathrm{Cl}_{2}\right]_{2}$ was prepared by modification of a previously published procedure. ${ }^{25}\left[\mathrm{Os}\left(\eta^{6}-p\right.\right.$-cym)(acetone $\left.)_{3}\right]\left(\mathrm{CF}_{3} \mathrm{SO}_{3}\right)_{2}$ and $\left[\mathrm{Os}\left(\eta^{6}-p\right.\right.$-cym $\left.)(\text { methanol })_{3}\right]\left(\mathrm{CF}_{3} \mathrm{SO}_{3}\right)_{2}$ were obtained in a similar manner as $\left[\mathrm{Ru}\left(\eta^{6}-p\right.\right.$-cym)(acetone $\left.)_{3}\right]\left(\mathrm{CF}_{3} \mathrm{SO}_{3}\right)_{2} .{ }^{24} \mathrm{H}-\mathrm{NMR}$ spectra were recorded on a Bruker AM360 FT-NMR instrument at room temperature in $\left(\mathrm{CD}_{3}\right)_{2} \mathrm{SO}$, and referenced to TMS as the internal standard. IR spectra ( $\mathrm{KBr}$ pellets) were recorded on a Perkin Elmer FTIR Paragon 1000 PC instrument, and ESI-MS spectra (methanolic solutions) with a Bruker micrOTOF-Q 9 instrument in the positive mode. Elemental analyses $(\mathrm{C}, \mathrm{H}, \mathrm{N}, \mathrm{S})$ were conducted on an Elementar Variomicro Cube instrument at the Department of Organic Chemistry, Debrecen University, Hungary. DMF, all cell culture reagents and media were purchased from Sigma-Aldrich Ireland, Ltd, unless otherwise stated.

\subsection{Crystal structure analysis}

Diffraction intensity data collection was carried out on a BrukerNonius MACH3 or an Agilent-Oxford SuperNova diffractometer using graphite-monochromated Mo-K $\alpha$ radiation $(\lambda=0.71073 \AA)$. The structures were solved by the SIR-92 program ${ }^{26}$ and refined by full-matrix least-squares method on $F^{2}$, with all non-hydrogen atoms refined with anisotropic thermal parameters using the SHELXL-97 package, ${ }^{27}$ publication material was prepared with the WINGX- suite. ${ }^{28}$ All hydrogen atoms were located geometrically, and refined using the riding model. Crystallographic and experimental details are summarized in Table 1.

\subsection{Synthesis of complexes}

[Os( $\left.\left.\eta^{6}-p-\mathbf{c y m}\right) \mathrm{Cl}_{2}\right]_{2}$ (1). To a solution of $\mathrm{H}_{2}\left[\mathrm{OsCl}_{6}\right](3.19 \mathrm{~g}$, $7.87 \mathrm{mmol})$ in dry EtOH $(35 \mathrm{~mL}) \alpha$-terpinene $(12.8 \mathrm{~mL}, 78.4 \mathrm{mmol})$ was added and the reaction mixture was refluxed under $\mathrm{N}_{2}$ for $50 \mathrm{~h}$ and allowed to stand at $4{ }^{\circ} \mathrm{C}$ for $24 \mathrm{~h}$. The crude orange product was filtered and recrystallised in EtOH ( $2.07 \mathrm{~g}, 2.61 \mathrm{mmol}, 66 \%)$. Calcd. for $\mathrm{C}_{20} \mathrm{H}_{28} \mathrm{Cl}_{4} \mathrm{Os}_{2}$ : C, 30.38; $\mathrm{H}, 3.57$; found $\%$ C, $29.88 ; \mathrm{H}, 3.37 .{ }^{1} \mathrm{H}-$ NMR (360 MHz, $d_{6}$-DMSO, $298 \mathrm{~K}$, TMS, $\mathrm{s}=$ singlet, $\mathrm{d}=$ doublet, $\mathrm{t}$ $=$ triplet, $\mathrm{h}=$ heptet, $\mathrm{m}=$ multiplet $): \delta=1.19\left[\mathrm{~d}, 6 \mathrm{H},-\mathrm{CH}\left(\mathrm{CH}_{3}\right)_{2}, J=\right.$ $7.02 \mathrm{~Hz}], 2.13\left[\mathrm{~s}, 3 \mathrm{H},-\mathrm{CH}_{3}\right], 2.74\left[\mathrm{~h}, 1 \mathrm{H},-\mathrm{CH}\left(\mathrm{CH}_{3}\right)_{2}, J=7.02 \mathrm{~Hz}\right]$, 5.99 [d, 2H, $\left.\operatorname{Ar}(-H)_{2} J=5.96 \mathrm{~Hz}\right], 6.07$ [d, $\left.2 \mathrm{H}, \operatorname{Ar}(-H)_{2} J=5.96 \mathrm{~Hz}\right]$. IR (KBr): $v_{\max } / \mathrm{cm}^{-1}=3050 \mathrm{~s}(\mathrm{Ar}-\mathrm{H}), 3042 \mathrm{~s}(\mathrm{Ar}-\mathrm{H}), 2960$ vs. $(\mathrm{C}-$ H), $2924 \mathrm{~s}, 2868 \mathrm{~s}(\mathrm{C}-\mathrm{H}), 1470 \mathrm{~s}, 1448 \mathrm{~s}(\mathrm{C}-\mathrm{C}), 1388 \mathrm{~s}, 1362 \mathrm{~m}$, $1054 \mathrm{~s}, 878 \mathrm{~s}$. MS (ESI-TOF): $m / z(\%)=743.1921(100)\left[\mathrm{Os}_{2}\left(\eta^{6}-\right.\right.$ $p$-cym $\left.)_{2}(\mu-\mathrm{OMe})_{3}\right]^{+}, 751.0937(60)\left[\mathrm{Os}_{2}\left(\eta^{6}-p \text {-cym }\right)_{2}(\mu-\mathrm{OMe})(\mu-\mathrm{Cl})_{2}\right]^{+}$, $729.1765(5)\left[\mathrm{Os}_{2}\left(\eta^{6}-p-c y m\right)_{2}(\mu-\mathrm{OMe})_{2}(\mu-\mathrm{OH})\right]^{+}$.

[Os( $\boldsymbol{\eta}^{6}$-p-cym)(acetone $\left.)_{3}\right]\left(\mathrm{CF}_{3} \mathrm{SO}_{3}\right)_{2}$ (2). $\mathrm{AgCF}_{3} \mathrm{SO}_{3}(102.8 \mathrm{mg}$, $0.4 \mathrm{mmol})$ was added to a solution of $1(79.1 \mathrm{mg}, 0.1 \mathrm{mmol})$ in dry acetone $(8 \mathrm{~mL})$ giving a yellow solution and an immediate precipitate of $\mathrm{AgCl}$. The mixture was protected from light, stirred at r.t. for $30 \mathrm{~min}$, and filtered to remove $\mathrm{AgCl}$. The resulting solution was evaporated, and the brown-yellow thick oil was dried in vacuo and used in subsequent steps.

$\left[\mathrm{Os}\left(\eta^{6}-p\right.\right.$-cym $\left.)(\text { methanol })_{3}\right]\left(\mathrm{CF}_{3} \mathrm{SO}_{3}\right)_{2}$ (3). It was obtained as $\mathbf{2}$ using dry $\mathrm{MeOH}(10 \mathrm{~mL})$. The resulting thick oil was used in subsequent steps.
[Os $\left(\boldsymbol{\eta}^{6}-\boldsymbol{p}\right.$-cym $)(\boldsymbol{\mu} \text {-meaha) }]_{2}\left(\mathbf{C F}_{3} \mathbf{S O}_{3}\right)_{2}$ (4). MeahaH (35.60 mg, $0.4 \mathrm{mmol})$ and $\mathrm{NaOMe}(21.60 \mathrm{mg}, 0.4 \mathrm{mmol})$ were added to a solution of $3(143.6 \mathrm{mg}, 0.2 \mathrm{mmol})$ in dry $\mathrm{MeOH}(15 \mathrm{~mL})$, protected from light and stirred at r.t. for $2.5 \mathrm{~h}$ under $\mathrm{N}_{2}$. The solvent was removed by rotary evaporation, and the residue was extracted with $\mathrm{CH}_{2} \mathrm{Cl}_{2}$ followed by the addition of diethyl ether. Slow evaporation at $-20{ }^{\circ} \mathrm{C}$ resulted in the formation of winered crystals. The complex was filtered washed with diethyl ether and dried under vacuum. Yield: $32.6 \mathrm{mg}(0.029 \mathrm{mmol}, 29 \%)$. Calcd. for $\mathrm{C}_{14} \mathrm{H}_{20} \mathrm{~F}_{3} \mathrm{NO}_{5} \mathrm{OsS}$ : C, 29.94; H, 3.59; N, 2.49; $\mathrm{S}, 5.71$, found C, 29.75; H, 3.41; N, 2.39; S, 5.63. ${ }^{1} \mathrm{H}-\mathrm{NMR}(360 \mathrm{MHz}$, $d_{6}$-DMSO, $298 \mathrm{~K}$, TMS): 1.22 [d, $6 \mathrm{H},-\mathrm{CH}\left(\mathrm{CH}_{3}\right)_{2}, J=6.67 \mathrm{~Hz}$ ], $2.11\left[\mathrm{~s}, 3 \mathrm{H},-\mathrm{CH}_{3}\right], 2.21\left[\mathrm{~s}, 3 \mathrm{H},-\mathrm{CH}_{3}\right], 2.65\left[\mathrm{~h}, 1 \mathrm{H},-\mathrm{CH}\left(\mathrm{CH}_{3}\right)_{2}\right.$, $J=6.67 \mathrm{~Hz}], 3.30\left[\mathrm{~s}, 3 \mathrm{H}, \mathrm{N}\left(-\mathrm{CH}_{3}\right)\right], 6.04\left[\mathrm{~d}, 2 \mathrm{H}, \operatorname{Ar}\left(-\mathrm{H}_{2}\right.\right.$, $J=5.61 \mathrm{~Hz}], 6.19\left[\mathrm{~d}, 2 \mathrm{H}, \operatorname{Ar}(-H)_{2}, J=5.61 \mathrm{~Hz}\right] . \mathrm{IR}(\mathrm{KBr})$ : $v_{\max } / \mathrm{cm}^{-1}=3062 \mathrm{w}(\mathrm{Ar}-\mathrm{H}), 2968 \mathrm{~m}(\mathrm{C}-\mathrm{H}), 2874 \mathrm{w}(\mathrm{C}-\mathrm{H}), 1622 \mathrm{~m}$ $(\mathrm{C}=\mathrm{O}), 1604 \mathrm{~s}(\mathrm{C}-\mathrm{C}), 1412 \mathrm{~s}, 1274$ vs. (triflate), 1260 vs. (triflate), $1158 \mathrm{~s}, 1030 \mathrm{~s}$ (triflate), $886 \mathrm{~s}, 638 \mathrm{~s}$ (triflate). MS (ESI-TOF): $\mathrm{m} / \mathrm{z}$ $(\%)=414.112(100)\left[\mathrm{M}-2 \mathrm{CF}_{3} \mathrm{SO}_{3}\right]^{2+}$.

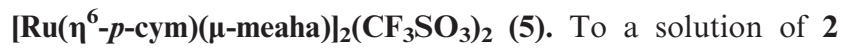
$(169.84 \mathrm{mg}, 0.240 \mathrm{mmol})$ in dry $\mathrm{MeOH}(5 \mathrm{~mL})$ meahaH (21.36 mg, $0.24 \mathrm{mmol}$ ) and $\mathrm{NaOMe}(12.82 \mathrm{mg}, 0.240 \mathrm{mmol})$ was added and stirred for $4 \mathrm{~h}$ at r.t. under $\mathrm{N}_{2}$. The solvent was removed by rotary evaporation, and the residue extracted with $\mathrm{CH}_{2} \mathrm{Cl}_{2}$. After filtering the sodium triflate on cotton wool, diisopropyl ether was added to the filtrate. On cooling at $-20^{\circ} \mathrm{C}$ for $24 \mathrm{~h}$, an orange microcrystalline solid was formed. The complex was filter washed with diethyl ether and dried under vacuum. Yield: $56 \mathrm{mg}$ $(0.059 \mathrm{mmol}, 49 \%)$. Crystals of 5 suitable for X-ray structural analysis were obtained by slow diffusion of layered diethyl ether in an acetone solution of the complex at $-20{ }^{\circ} \mathrm{C}$. Calcd. for $\mathrm{C}_{14} \mathrm{H}_{20} \mathrm{~F}_{3} \mathrm{NO}_{5}$ RuS: C, 35.59; H, 4.27; N, 2.96; S, 6.79, found $\mathrm{C}$, $35.55 ; \mathrm{H}, 4.23$; N, 2.89; S, 6.02. ${ }^{1} \mathrm{H}-\mathrm{NMR}\left(360 \mathrm{MHz}, d_{6}\right.$-DMSO, $298 \mathrm{~K}, \mathrm{TMS}): \delta=1.23$ [d, $\left.6 \mathrm{H},-\mathrm{CH}\left(\mathrm{CH}_{3}\right)_{2}\right], \delta=2.02\left[\mathrm{~s}, 3 \mathrm{H},-\mathrm{CH}_{3}\right.$ ], $\delta=2.14\left[\mathrm{~s}, 3 \mathrm{H},-\mathrm{CH}_{3}\right], \delta=2.74\left[\mathrm{~h}, 1 \mathrm{H},-\mathrm{CH}\left(\mathrm{CH}_{3}\right)_{2}\right], \delta=3.26[\mathrm{~s}$, $\left.3 \mathrm{H}, \mathrm{N}\left(-\mathrm{C} H_{3}\right)\right], \delta=5.70\left[\mathrm{~d}, 2 \mathrm{H}, \operatorname{Ar}(-H)_{2}\right], \delta=5.88\left[\mathrm{~d}, 2 \mathrm{H}, \operatorname{Ar}(-H)_{2}\right]$. IR $(\mathrm{KBr}): v_{\text {max }} / \mathrm{cm}^{-1}=3069 \mathrm{w}(\mathrm{Ar}-\mathrm{H}), 2965 \mathrm{~m}(\mathrm{C}-\mathrm{H}), 1614 \mathrm{~s}$ $(\mathrm{C}=\mathrm{O}), 1472 \mathrm{w}, 1437 \mathrm{w}, 1263 \mathrm{vs.}$ (triflate), 1227 vs. (triflate), $1165 \mathrm{~s}$, 1033 vs. (S-O), $640 \mathrm{w}, 518 \mathrm{w}$ (triflate). MS (ESI-TOF): $\mathrm{m} / z(\%)=$ $324.058(100)\left[\mathrm{M}-2 \mathrm{CF}_{3} \mathrm{SO}_{3}\right]^{2+}$.

[Os( $\boldsymbol{\eta}^{\mathbf{6}}-\boldsymbol{p}$-cym)(meaha)Cl] (6). 1 (79.17 mg, $\left.0.1 \mathrm{mmol}\right)$ in dry $\mathrm{MeOH}(15 \mathrm{~mL})$ was protected from light, stirred at r.t. for 30 min under $\mathrm{N}_{2}$ and meahaH ( $35.60 \mathrm{mg}, 0.4 \mathrm{mmol}$ ) and $\mathrm{NaOMe}$ (21.60 $\mathrm{mg}, 0.4 \mathrm{mmol}$ ) were added. The reaction mixture was stirred for a further $4 \mathrm{~h}$. The solvent was removed by rotary evaporation, and the residue extracted with $\mathrm{CH}_{2} \mathrm{Cl}_{2}$. After filtering $\mathrm{NaCl}$, the solution was evaporated, and the oily residue was dissolved in acetone and diethyl ether was added. On cooling at $-20^{\circ} \mathrm{C}$ for $24 \mathrm{~h}$, yellow crystals were formed and the complex was filtered washed with diethyl ether and dried under vacuum. Yield: $48 \mathrm{mg}(0.107 \mathrm{mmol}, 54 \%)$. Crystals of 6 suitable for X-ray structural analysis were obtained by slow diffusion of layered diisopropyl ether in an acetone solution of the complex at $-20^{\circ} \mathrm{C}$. Calcd. for $\mathrm{C}_{13} \mathrm{H}_{20} \mathrm{ClNO}_{2} \mathrm{Os}: \mathrm{C}, 34.85 ; \mathrm{H}, 4.50 ; \mathrm{N}, 3.13$; found $\% \mathrm{C}$, 34.24; H, 4.17; N, 2.95. ${ }^{1} \mathrm{H}-\mathrm{NMR}\left(360 \mathrm{MHz}, d_{6}\right.$-DMSO, $298 \mathrm{~K}$, TMS): $\delta=1.20\left[\mathrm{~d}, 6 \mathrm{H},-\mathrm{CH}\left(\mathrm{CH}_{3}\right)_{2}, J=6.67 \mathrm{~Hz}\right], 1.99$ [s, 3H, $(-$ $\left.\left.\mathrm{CH}_{3}\right)\right], 2.15\left[\mathrm{~s}, 3 \mathrm{H},-\mathrm{CH}_{3}\right], 2.50\left[\mathrm{~h}, 1 \mathrm{H},-\mathrm{CH}\left(\mathrm{CH}_{3}\right)_{2}\right], 3.20[\mathrm{~s}, 3 \mathrm{H}$, 
$\mathrm{N}\left(-\mathrm{CH}_{3}\right)$ ], 5.64 [s, 2H, $\left.\operatorname{Ar}(-H)_{2}\right], 5.89$ [s, 2H, $\left.\operatorname{Ar}(-H)_{2}\right] . \mathrm{IR}(\mathrm{KBr})$ : $v_{\text {max }} / \mathrm{cm}^{-1}=3056 \mathrm{w}$, and $3040 \mathrm{~m}(\mathrm{Ar}-\mathrm{H}), 2958 \mathrm{~m}(\mathrm{C}-\mathrm{H}), 2922 \mathrm{~m}$, $2870 \mathrm{~m}(\mathrm{C}-\mathrm{H}), 1618 v \mathrm{~s} .(\mathrm{C}=\mathrm{O}), 1468 \mathrm{~s}, 1434 \mathrm{~s}, 1162 \mathrm{~m}, 752 \mathrm{~s}, 654 \mathrm{~s}$, 592 s. MS (ESI-TOF): $m / z(\%)=414.113(100)[\mathrm{M}-\mathrm{Cl}]^{+}$.

$\left[\mathbf{R u}\left(\boldsymbol{\eta}^{\mathbf{6}}-\boldsymbol{p}\right.\right.$-cym)(meaha)Cl] (7). It was obtained in an analogous manner as 6 using $70.14 \mathrm{mg}(0.115 \mathrm{mmol})\left[\mathrm{Ru}\left(\eta^{6}-p-c y m\right) \mathrm{Cl}_{2}\right]_{2}$, $41.08 \mathrm{mg}(0.458 \mathrm{mmol}) \mathrm{meahaH}$ and $24.75 \mathrm{mg}(0.458 \mathrm{mmol})$ NaOMe. Yield: $47 \mathrm{mg}(0.131 \mathrm{mmol}, 57 \%)$. Calcd. for $\mathrm{C}_{13} \mathrm{H}_{20} \mathrm{ClNO}_{2} \mathrm{Ru}$ : C, 43.51; H, 5.62; N, 3.90; found C, 43.22; $\mathrm{H}, 5.61$; N, 4.13. ${ }^{1} \mathrm{H}-\mathrm{NMR}$ (360 MHz, $d_{6}$-DMSO, $\left.298 \mathrm{~K}, \mathrm{TMS}\right)$ : $\delta=1.22\left[\mathrm{~d}, 6 \mathrm{H},-\mathrm{CH}\left(\mathrm{CH}_{3}\right)_{2}\right], \delta=1.85\left[\mathrm{~s}, 3 \mathrm{H},-\mathrm{CH}_{3}\right], \delta=2.10$ $\left[\mathrm{s}, 3 \mathrm{H},-\mathrm{CH}_{3}\right], \delta=2.69\left[\mathrm{~h}, 1 \mathrm{H},-\mathrm{CH}\left(\mathrm{CH}_{3}\right)_{2}\right], \delta=3.09[\mathrm{~s}, 3 \mathrm{H}$, $\left.\mathrm{N}\left(-\mathrm{CH}_{3}\right)\right], \delta=5.22\left[\mathrm{~s}, 2 \mathrm{H}, \operatorname{Ar}(-H)_{2}\right], \delta=5.50\left[\mathrm{~s}, 2 \mathrm{H}, \operatorname{Ar}(-H)_{2}\right]$. IR $(\mathrm{KBr}): v_{\max } / \mathrm{cm}^{-1}=3060 \mathrm{w}, 3040 \mathrm{w}$, and $3026 \mathrm{w}(\mathrm{Ar}-\mathrm{H}), 2960 \mathrm{~m}$ $(\mathrm{C}-\mathrm{H}), 2928 \mathrm{~m}(\mathrm{C}-\mathrm{H}), 1612$ vs. $(\mathrm{C}=\mathrm{O}), 1468 \mathrm{~s}, 1432 \mathrm{~s}, 954 \mathrm{~m}, 752 \mathrm{~s}$ $652 \mathrm{~s}, 568$ s. MS (ESI-TOF): $m / z(\%)=324.058(100)[\mathrm{M}-\mathrm{Cl}]^{+}$.

[Os( $\boldsymbol{\eta}^{\mathbf{6}}$-p-cym)(phebha)Cl] (8). 1 (79.17 mg, $\left.0.1 \mathrm{mmol}\right)$ in dry $\mathrm{MeOH}(15 \mathrm{~mL})$ was protected from light, and stirred at r.t. for $30 \mathrm{~min}$ under $\mathrm{N}_{2}$. PhebhaH (85.20 mg, $\left.0.4 \mathrm{mmol}\right)$ and $\mathrm{NaOMe}$ (21.60 mg, $0.4 \mathrm{mmol}$ ) were then added. The reaction mixture was again stirred and after $1 \mathrm{~h}$, yellow crystals of $\mathbf{8}$ appeared. The complex was filtered, washed with diethyl ether and dried under vacuum. Yield: $51.49 \mathrm{mg}(0.090 \mathrm{mmol}, 45 \%)$. Calcd. for $\mathrm{C}_{23} \mathrm{H}_{24} \mathrm{ClNO}_{2}$ Os: C, 48.28; $\mathrm{H}, 4.23$; N, 2.45; found C, 48.10; H, 3.90; N, 2.49. ${ }^{1} \mathrm{H}-\mathrm{NMR}$ (360 MHz, $d_{6}$-DMSO, $298 \mathrm{~K}$, TMS): $\delta=1.23\left[\mathrm{~d}, 6 \mathrm{H},-\mathrm{CH}\left(\mathrm{CH}_{3}\right)_{2}\right], 2.25\left[\mathrm{~s}, 3 \mathrm{H},-\mathrm{CH}_{3}\right], 2.65[\mathrm{~h}$, $\left.1 \mathrm{H},-\mathrm{CH}\left(\mathrm{CH}_{3}\right)_{2}\right], 5.80\left[\mathrm{~s}, 2 \mathrm{H}, \operatorname{Ar}(-H)_{2}\right], 6.09$ [q, 2H, $\operatorname{Ar}(-H)_{2}$ ], 7.13-7.38 [m, 10H, $\left.\left(-\mathrm{C}_{6} H_{5}\right)_{2}\right]$. IR $(\mathrm{KBr}): v_{\max } / \mathrm{cm}^{-1}=3060 \mathrm{~m}$ $(\mathrm{Ar}-\mathrm{H}), 2962 \mathrm{~m}(\mathrm{C}-\mathrm{H}), 2922 \mathrm{~m}(\mathrm{C}-\mathrm{H}), 2870 \mathrm{~m}(\mathrm{C}-\mathrm{H}), 1584 \mathrm{vs}$, 1548 vs, 1498 s, 1430 vs. (ring C-C), 1010 s, 936 s, 772 s, 694 vs, 448 m. MS (ESI-TOF): $m / z(\%)=538.150(100)[\mathrm{M}-\mathrm{Cl}]^{+}$.

[Ru( $\left.\left.\eta^{6}-p-c y m\right)(p h e b h a) C l\right]$ (9). To a solution of $\left[\mathrm{Ru}\left(\eta^{6}-\right.\right.$ p-cym) $\left.\mathrm{Cl}_{2}\right]_{2}(70.19 \mathrm{mg}, 0.115 \mathrm{mmol})$ in dry $\mathrm{MeOH}(5 \mathrm{~mL})$ phebhaH (97.57 mg, $0.458 \mathrm{mmol}$ ) and $\mathrm{NaOMe}(24.75 \mathrm{mg}$, $0.458 \mathrm{mmol}$ ) was added, and stirred for $2 \mathrm{~h}$ at r.t. under $\mathrm{N}_{2}$. The solvent was removed by rotary evaporation, and the residue was extracted with $\mathrm{CH}_{2} \mathrm{Cl}_{2}$. After filtering $\mathrm{NaCl}$, the solution was left to evaporate slowly. Brown coloured crystals were filtered, washed with diethyl ether and dried under vacuum. Yield: $59 \mathrm{mg}$ (0.122 mmol, 53\%). Calcd. for $\mathrm{C}_{23} \mathrm{H}_{24} \mathrm{ClNO}_{2} \mathrm{Ru}$ : C, 57.20; $\mathrm{H}$, $5.01 ; \mathrm{N}, 2.90$; found $\mathrm{C}, 56.66 ; \mathrm{H}, 5.02 ; \mathrm{N}, 2.90$. The obtained crystals were found to be directly suitable for X-ray structural analysis. ${ }^{1} \mathrm{H}-\mathrm{NMR}$ (360 MHz, $d_{6}$-DMSO, $298 \mathrm{~K}, \mathrm{TMS}$ ): $\delta=1.30$ [d, $\left.6 \mathrm{H},-\mathrm{CH}\left(\mathrm{CH}_{3}\right)_{2}, J=6.58 \mathrm{~Hz}\right], \delta=2.19\left[\mathrm{~s}, 3 \mathrm{H},-\mathrm{CH}_{3}\right], \delta=2.80$ $\left[\mathrm{h}, 1 \mathrm{H},-\mathrm{CH}\left(\mathrm{CH}_{3}\right)_{2}\right], \delta=5.39\left[\mathrm{~d}, 2 \mathrm{H}, \operatorname{Ar}(-H)_{2}, J=5.94 \mathrm{~Hz}\right]$, $\delta=5.69\left(\mathrm{~d}, 2 \mathrm{H}, \operatorname{Ar}(-H)_{2}, J=6.80 \mathrm{~Hz}\right], \delta=7.05-7.24[\mathrm{~m}, 10 \mathrm{H}$, $\left.\left(-\mathrm{C}_{6} H_{5}\right)_{2}\right]$. IR $(\mathrm{KBr}): v_{\max } / \mathrm{cm}^{-1}=3056(\mathrm{Ar}-\mathrm{H}), 2962(\mathrm{C}-\mathrm{H})$, 2871, 1584 s, 1555 s, 1429, 1148, 1011, 936, 773, 694. MS (ESITOF): $m / z(\%)=448,086(100)[\mathrm{M}-\mathrm{Cl}]^{+}$.

[Os( $\boldsymbol{\eta}^{\mathbf{6}}$-p-cym)(meaha)(py)] $\mathbf{C F}_{\mathbf{3}} \mathbf{S O}_{\mathbf{3}}$ (10). MeahaH (35.60 mg, $0.4 \mathrm{mmol}), \mathrm{NaOMe}(21.60 \mathrm{mg}, 0.4 \mathrm{mmol})$ and pyridine $(0.016 \mathrm{~mL}$, $0.2 \mathrm{mmol})$ were added to a solution of $2(159.48 \mathrm{mg}, 0.2 \mathrm{mmol})$ in dry $\mathrm{MeOH}(15 \mathrm{~mL})$, protected from light and stirred at r.t. for $4 \mathrm{~h}$ under $\mathrm{N}_{2}$. The solvent was removed by rotary evaporation, and the residue was dissolved in $\mathrm{CH}_{2} \mathrm{Cl}_{2}$, filtered and evaporated. The oily residue was redissolved in acetone and dipropyl ether was added. Slow evaporation at $-20{ }^{\circ} \mathrm{C}$ resulted in the formation of yellow crystals. The hygroscopic complex was quickly filtered washed with diisopropyl ether and dried under vacuum. Yield: $81.1 \mathrm{mg}$ (126.4 mmol, 63\%). Calcd. for $\mathrm{C}_{19} \mathrm{H}_{25} \mathrm{~F}_{3} \mathrm{~N}_{2} \mathrm{O}_{5} \mathrm{OsS}: \mathrm{C}$, 35.62; H, 3.93; N, 4.37; S, 5.00. Found C, 35.12; H, 3.70; N, 4.39; $\mathrm{S}, 4.97 .{ }^{1} \mathrm{H}-\mathrm{NMR}\left(360 \mathrm{MHz}, d_{6}\right.$-DMSO, $\left.298 \mathrm{~K}, \mathrm{TMS}\right): \delta=1.20[\mathrm{~d}$, $\left.6 \mathrm{H},-\mathrm{CH}\left(\mathrm{CH}_{3}\right)_{2}, J=7.06 \mathrm{~Hz}\right], 1.92\left[\mathrm{~s}, 3 \mathrm{H},-\mathrm{CH}_{3}\right], 2.01[\mathrm{~s}$, $\left.3 \mathrm{H},-\mathrm{CH}_{3}\right], 2.57\left[\mathrm{~h}, 1 \mathrm{H},-\mathrm{CH}\left(\mathrm{CH}_{3}\right)_{2}, J=7.06 \mathrm{~Hz}\right], 3.16[\mathrm{~s}, 3 \mathrm{H}$, $\left.\mathrm{N}\left(-\mathrm{CH}_{3}\right)\right], 5.81\left[\mathrm{t}, 2 \mathrm{H}, \operatorname{Ar}(-\mathrm{H})_{2}\right], 6.07\left[\mathrm{dd}, 2 \mathrm{H}, \operatorname{Ar}(-H)_{2}\right], 7.57[\mathrm{t}$, $\left.2 \mathrm{H}, \mathrm{py}(-\mathrm{H})_{2}\right], 8.01[\mathrm{t}, 1 \mathrm{H}, \mathrm{py}-\mathrm{H}], 8.47$ [d, 2H, py $\left.(-H)_{2}\right]$. IR $(\mathrm{KBr})$ : $v_{\max } / \mathrm{cm}^{-1}=3066 \mathrm{~s}(\mathrm{Ar}-\mathrm{H}), 2968 \mathrm{~s}(\mathrm{C}-\mathrm{H}), 1620$ vs. $(\mathrm{C}=\mathrm{O}), 1450$ vs. (py), 1284 vs, 1226 vs. (triflate), 1150 vs, 1030 vs. (S-O), 754 vs, 702 vs. (py), $636 \mathrm{vs,} 518 \mathrm{~s}$ (triflate). MS (ESI-TOF): $m / z(\%)=414.116$ (100) $\left[\mathrm{M}-\mathrm{CF}_{3} \mathrm{SO}_{3}-\mathrm{Py}\right]^{+}$.

$\left[\mathbf{R u}\left(\eta^{6}-p-c y m\right)(\right.$ meaha)(py) $] \mathrm{CF}_{3} \mathbf{S O}_{3}$ (11). It was obtained in an analogous manner as $\mathbf{1 0}$ using $141.53 \mathrm{mg}(0.20 \mathrm{mmol}) \mathbf{2}, 35.60 \mathrm{mg}$ (0.40 mmol) meahaH, $21.60 \mathrm{mg}(0.40 \mathrm{mmol}) \mathrm{NaOMe}$ and $0.016 \mathrm{~mL}(0.20 \mathrm{mmol})$ pyridine in dry $\mathrm{MeOH}(6 \mathrm{~mL})$. Yield: $34.22 \mathrm{mg}(0.062 \mathrm{mmol}, 31 \%)$. Calcd. for $\mathrm{C}_{19} \mathrm{H}_{25} \mathrm{~F}_{3} \mathrm{~N}_{2} \mathrm{O}_{5} \mathrm{RuS}$ : C, 41.38; H, 4.57; N, 5.08; S, 5.81. Found C, 41.30; H, 4.62; N, 5.02; $\mathrm{S}, 5.85 .{ }^{1} \mathrm{H}-\mathrm{NMR}\left(360 \mathrm{MHz}, d_{6}\right.$-DMSO, $\left.298 \mathrm{~K}, \mathrm{TMS}\right): \delta=1.22[\mathrm{~d}$, $\left.6 \mathrm{H},-\mathrm{CH}\left(\mathrm{CH}_{3}\right)_{2}, J=6.94 \mathrm{~Hz}\right], 1.80\left[\mathrm{~s}, 3 \mathrm{H},-\mathrm{CH}_{3}\right], 1.96[\mathrm{~s}$, $\left.3 \mathrm{H},-\mathrm{CH}_{3}\right], 2.68\left[\mathrm{~h}, 1 \mathrm{H},-\mathrm{CH}\left(\mathrm{CH}_{3}\right)_{2}, J=6.94 \mathrm{~Hz}\right], 3.08[\mathrm{~s}, 3 \mathrm{H}$, $\left.\mathrm{N}\left(-\mathrm{CH}_{3}\right)\right], 5.55\left[\mathrm{t}, 2 \mathrm{H}, \operatorname{Ar}(-H)_{2}\right], 5.81\left[\mathrm{dd}, 2 \mathrm{H}, \operatorname{Ar}(-H)_{2}\right], 7.57[\mathrm{t}, 2 \mathrm{H}$, py $\left.(-H)_{2}\right], 8.03[\mathrm{t}, 1 \mathrm{H}$, py- $H], 8.52\left[\mathrm{~d}, 2 \mathrm{H}, \operatorname{py}(-H)_{2}\right]$. IR $(\mathrm{KBr})$ : $v_{\max } / \mathrm{cm}^{-1}=3068 \mathrm{~m}(\mathrm{Ar}-\mathrm{H}), 2968 \mathrm{~m}(\mathrm{C}-\mathrm{H}), 2942 \mathrm{~m}(\mathrm{C}-\mathrm{H}), 2876 \mathrm{w}$ $(\mathrm{C}-\mathrm{H}), 1608 \mathrm{~s}(\mathrm{C}=\mathrm{O}), 1470 \mathrm{~s}, 1448 \mathrm{~s}$ (py), $1424 \mathrm{~s}, 1404 \mathrm{~m}, 1276$ vs. (triflate), $1224 \mathrm{~s}$ (triflate), $1030 \mathrm{vs}$. (triflate), $952 \mathrm{~m}, 752 \mathrm{~s}, 638 \mathrm{vs,}$ $572 \mathrm{~s}, 518 \mathrm{~m}$ (triflate). MS (ESI-TOF): $\mathrm{m} / z(\%)=324.058$ (100) $\left[\mathrm{M}-\mathrm{CF}_{3} \mathrm{SO}_{3}-\mathrm{Py}\right]^{+}$.

$\left[\mathbf{R u}\left(\boldsymbol{\eta}^{\mathbf{6}}-\boldsymbol{p}\right.\right.$-cym)(bha)(py)] $\mathrm{CF}_{3} \mathrm{SO}_{3}$ (12). It was obtained in an analogous manner as $\mathbf{1 0}$ using $290.14 \mathrm{mg}$ (0.410 mmol) 2, $54.80 \mathrm{mg}$ (0.40 mmol) bhaH, $21.60 \mathrm{mg}(0.40 \mathrm{mmol}) \mathrm{NaOMe}$ and $0.032 \mathrm{~mL}(0.40 \mathrm{mmol})$ pyridine. Yield: $149 \mathrm{mg}(0.341 \mathrm{mmol}$, $83 \%$ ). Calcd. for $\mathrm{C}_{23} \mathrm{H}_{25} \mathrm{~F}_{3} \mathrm{~N}_{2} \mathrm{O}_{5} \mathrm{RuS}$ : C, 46.07; H, 4.20; N, 4.67; S, 5.35. Found C, 46.13; H, 4.23; N, 4.70; S, 5.28. Crystals of 12 suitable for X-ray structural analysis were obtained by slow diffusion of layered diethyl ether in an acetone solution of the complex at $-20{ }^{\circ} \mathrm{C}$. ${ }^{1} \mathrm{H}-\mathrm{NMR}\left(360 \mathrm{MHz}, d_{6}\right.$-DMSO, $298 \mathrm{~K}$, TMS): $\delta=1.27\left[\mathrm{~d}, 6 \mathrm{H},-\mathrm{CH}\left(\mathrm{CH}_{3}\right)_{2}, J=6.80 \mathrm{~Hz}\right], 2.06[\mathrm{~s}$, $\left.3 \mathrm{H},-\mathrm{CH}_{3}\right], 2.77\left[\mathrm{~h}, 1 \mathrm{H},-\mathrm{CH}\left(\mathrm{CH}_{3}\right)_{2}, J=6.80 \mathrm{~Hz}\right], 5.65[\mathrm{~d}, 2 \mathrm{H}$, $\left.\operatorname{Ar}(-H)_{2}\right], 5.92\left[\mathrm{~d}, 2 \mathrm{H}, \operatorname{Ar}(-H)_{2}\right], 7.40-7.58\left[\mathrm{~m}, 7 \mathrm{H},-\mathrm{C}_{6} H_{5}, 2 \mathrm{H}\right.$ of py $\left.(-H)_{2}\right], 7.97[\mathrm{t}, 1 \mathrm{H}$ py- $H], 8.58\left[\mathrm{~d}, 2 \mathrm{H}\right.$ py $\left.(-H)_{2}\right]$. IR $(\mathrm{KBr})$ : $v_{\text {max }} / \mathrm{cm}^{-1}=3184,3111$ and $3062(\mathrm{Ar}-\mathrm{H}), 2963(\mathrm{C}-\mathrm{H}), 1598$ $(\mathrm{C}=\mathrm{O}), 1507,1481,1448$ (py), 1294, 1224 (triflate), 1159, 1028 (triflate), 913, 763, 694 (triflate), 637, 567, 515 (triflate). MS (ESI-TOF): $m / z(\%)=372.053(100)\left[\mathrm{M}-\mathrm{CF}_{3} \mathrm{SO}_{3}-\mathrm{Py}\right]^{+}$.

[Os( $\boldsymbol{\eta}^{\mathbf{6}}$-p-cym)(phebha)(py)] $\mathbf{C F}_{3} \mathbf{S O}_{\mathbf{3}}$ (13). PhebhaH (170.70 mg, $0.8 \mathrm{mmol}), \mathrm{NaOMe}(42.50 \mathrm{mg}, 0.8 \mathrm{mmol})$ and pyridine $(0.063 \mathrm{~mL}$, $0.8 \mathrm{mmol})$ were added to a solution of $2(318.7 \mathrm{mg}, 0.4 \mathrm{mmol})$ in dry $\mathrm{MeOH}(10 \mathrm{~mL})$, protected from light, and stirred at r.t. for $3 \mathrm{~h}$ under $\mathrm{N}_{2}$. The solvent was removed by rotary evaporation, and the residue was dissolved in $\mathrm{CH}_{2} \mathrm{Cl}_{2}$ and then filtered. To this solution, acetone and diisopropyl ether was added. Slow evaporation at $-20{ }^{\circ} \mathrm{C}$ resulted in the formation of yellow crystals. The complex 
was filtered and washed with diisopropyl ether, and dried under vacuum. Yield: $69.9 \mathrm{mg}(0.091 \mathrm{mmol}, 23 \%)$. Calcd. for $\mathrm{C}_{29} \mathrm{H}_{29} \mathrm{~F}_{3} \mathrm{~N}_{2} \mathrm{O}_{5} \mathrm{OsS}$ : C, 45.54; H, 3.82; N, 3.66; S, 4.19. Found $\mathrm{C}$, 45.07; H, 3.57; N, 3.69; S, 4.13. ${ }^{1} \mathrm{H}-\mathrm{NMR}\left(360 \mathrm{MHz}, d_{6}\right.$-DMSO, $298 \mathrm{~K}, \mathrm{TMS}): \delta=1.29$ [d, $\left.6 \mathrm{H},-\mathrm{CH}\left(\mathrm{CH}_{3}\right)_{2}, J=7.02 \mathrm{~Hz}\right], 2.09$ [s, $3 \mathrm{H},-\mathrm{CH}_{3}$ ], $2.72\left[\mathrm{~h}, 1 \mathrm{H},-\mathrm{CH}\left(\mathrm{CH}_{3}\right)_{2}, J=7.02 \mathrm{~Hz}\right.$ ], 5.99 [dd, $2 \mathrm{H}$, $\left.\operatorname{Ar}(-H)_{2}\right], 6.27\left[\mathrm{dd}, 2 \mathrm{H}, \operatorname{Ar}(-H)_{2}\right], 6.74\left[\mathrm{~d}, 2 \mathrm{H}, \operatorname{Ar}(-\mathrm{H})_{2}\right], 7.04$ [d, $2 \mathrm{H}], 7.24-7.42\left[\mathrm{~m}, 6 \mathrm{H},\left(-\mathrm{C}_{6} H_{5}\right)\right], 7.65\left[\mathrm{t}, 2 \mathrm{H}, \mathrm{py}(-H)_{2}\right], 8.07[\mathrm{t}$, $1 \mathrm{H}, \mathrm{py}-H], 8.66\left[\mathrm{~d}, 2 \mathrm{H}, \mathrm{py}(-H)_{2}\right]$. IR $(\mathrm{KBr}): v_{\max } / \mathrm{cm}^{-1}=3104 \mathrm{w}$ (Ar-H), 3064 m (Ar-H), 2968 s (C-H), 2932 m, 2900 w (C-H), $2874 \mathrm{w}(\mathrm{C}-\mathrm{H}), 1564$ vs. $(\mathrm{C}=\mathrm{O}), 1432$ vs, 1274 vs. (triflate), $1146 \mathrm{vs,}$ 1031 vs. (triflate), 772 vs. (py), $696 \mathrm{vs,}, 638 \mathrm{vs}, 594 \mathrm{~s}, 516 \mathrm{~s}$ (triflate), $446 \mathrm{~m}$. MS (ESI-TOF): $m / z(\%)=538,147(100)\left[\mathrm{M}-\mathrm{CF}_{3} \mathrm{SO}_{3}-\mathrm{Py}\right]^{+}$.

$\left[\mathbf{R u}\left(\eta^{6}-p-c y m\right)\left(\right.\right.$ phebha)(py)] $\mathrm{CF}_{3} \mathbf{S O}_{3}$ (14). It was obtained in an analogous manner as $\mathbf{1 3}$ using $162,76 \mathrm{mg}(0.230 \mathrm{mmol}) \mathbf{2}$, $49.02 \mathrm{mg}$, (0.230 mmol) phebhaH, $12.42 \mathrm{mg}(0.230 \mathrm{mmol})$ $\mathrm{NaOMe}$ and $0.020 \mathrm{~mL}(0.230 \mathrm{mmol})$ pyridine in dry $\mathrm{MeOH}$ (5mL). Yield: $121 \mathrm{mg}(0.179 \mathrm{mmol}, 78 \%)$. The orange-brown crystals obtained of $\mathbf{1 4}$ were directly suitable for X-ray structural analysis. Calcd. for $\mathrm{C}_{29} \mathrm{H}_{29} \mathrm{~F}_{3} \mathrm{~N}_{2} \mathrm{O}_{5} \mathrm{RuS}$ : C, 51.55; $\mathrm{H}, 4.33$; $\mathrm{N}, 4.15 ; \mathrm{S}, 4.75$. Found $\mathrm{C}, 51.66 ; \mathrm{H}, 4.37 ; \mathrm{N}, 4.15 ; \mathrm{S}, 4.76$. ${ }^{1} \mathrm{H}-\mathrm{NMR}\left(360 \mathrm{MHz}, d_{6}\right.$-DMSO, $\left.298 \mathrm{~K}, \mathrm{TMS}\right): \delta=1.32[\mathrm{~d}$, $\left.6 \mathrm{H},-\mathrm{CH}\left(\mathrm{CH}_{3}\right)_{2}, J=6.94 \mathrm{~Hz}\right], 2.06\left[\mathrm{~s}, 3 \mathrm{H},-\mathrm{CH}_{3}\right], 2.82[\mathrm{~h}$, $\left.1 \mathrm{H},-\mathrm{CH}\left(\mathrm{CH}_{3}\right)_{2}, J=6.94 \mathrm{~Hz}\right], 5.68$ [d, 2H, $\left.\operatorname{Ar}(-\mathrm{H})_{2}\right], 5.98$ [d, $2 \mathrm{H}$, $\left.\operatorname{Ar}(-\mathrm{H})_{2}\right], 6.69-8.11\left[\mathrm{~m}, 15 \mathrm{H},\left(-\mathrm{C}_{6} H_{5}\right)_{2}\right.$, py $\left.(-H)_{5}\right]$. IR $(\mathrm{KBr})$ : $v_{\max } / \mathrm{cm}^{-1}=3108 \mathrm{w}(\mathrm{Ar}-\mathrm{H})$, and $3066 \mathrm{w}(\mathrm{Ar}-\mathrm{H}), 2966 \mathrm{~m}(\mathrm{C}-\mathrm{H})$, 2930 w (C-H), 2872 w (C-H), 1604 m, 1582 m, 1554 s, 1542 s, $1498 \mathrm{~s}, 1448 \mathrm{~s}$ (py), $1418 \mathrm{~s}, 1274 \mathrm{vs}$. (triflate), $1268 \mathrm{vs}, 1224 \mathrm{~s}$ (triflate), $1150 \mathrm{~s}, 1030$ vs. (triflate), $774 \mathrm{~s}$ (py), 698 s, 636 vs. , $596 \mathrm{vw}, 572 \mathrm{~m}, 516 \mathrm{~m}$ (triflate). MS (ESI-TOF): $\mathrm{m} / \mathrm{z}(\%)=$ 448,084 (100) $\left[\mathrm{M}-\mathrm{CF}_{3} \mathrm{SO}_{3}-\mathrm{Py}\right]^{+}$.

[Os( $\boldsymbol{\eta}^{\mathbf{6}}$-p-cym)(meaha)(py)]Cl (15). MeahaH (71.20 mg, $0.8 \mathrm{mmol})$, NaOMe (43.2 $\mathrm{mg}, 0.8 \mathrm{mmol})$ and pyridine $(0.032 \mathrm{~mL}, 0.4 \mathrm{mmol})$ were added to a solution of $\mathbf{1}$ (158.34 mg, $0.2 \mathrm{mmol})$ in dry $\mathrm{MeOH}(15 \mathrm{~mL})$, protected from light, and stirred at r.t. for $21 \mathrm{~h}$ under $\mathrm{N}_{2}$. The solvent was removed by rotary evaporation, and the residue was dissolved in $\mathrm{CH}_{2} \mathrm{Cl}_{2}$ and filtered. To this solution, diethyl ether and diisopropyl ether was added. Slow evaporation at $-20{ }^{\circ} \mathrm{C}$ resulted in the formation of brown crystals. The complex was filtered, washed with diethyl ether, and dried under vacuum. Yield: $26.9 \mathrm{mg}(0.051 \mathrm{mmol}, 13.0 \%)$. Calcd. for $\mathrm{C}_{18} \mathrm{H}_{25} \mathrm{ClN}_{2} \mathrm{O}_{2} \mathrm{Os}$ : C, 41.02; H, 4.78; N, 5.31. Found C, 40.79; H, 4.67; N, 5.39. ${ }^{1} \mathrm{H}-$ NMR (360 MHz, $d_{6}$-DMSO, $\left.298 \mathrm{~K}, \mathrm{TMS}\right): \delta=1.20[\mathrm{~d}, 6 \mathrm{H},-$ $\left.\left.\mathrm{CH}\left(\mathrm{CH}_{3}\right)_{2}\right), J=6.66 \mathrm{~Hz}\right], 1.92\left[\mathrm{~s}, 3 \mathrm{H},-\mathrm{CH}_{3}\right], 2.01\left[\mathrm{~s}, 3 \mathrm{H},-\mathrm{CH}_{3}\right]$, $2.58\left[\mathrm{~h}, 1 \mathrm{H},-\mathrm{CH}\left(\mathrm{CH}_{3}\right)_{2}, J=6.66 \mathrm{~Hz}\right], 3.16\left[\mathrm{~s}, 3 \mathrm{H},=\mathrm{N}\left(-\mathrm{CH}_{3}\right)\right]$, $5.81\left[\mathrm{t}, 2 \mathrm{H}, \operatorname{Ar}(-H)_{2}\right], 6.07\left[\mathrm{dd}, 2 \mathrm{H}, \operatorname{Ar}(-H)_{2}\right], 7.58\left[\mathrm{t}, 2 \mathrm{H}, \operatorname{py}(-H)_{2}\right]$, $8.01[\mathrm{t}, 1 \mathrm{H}, \mathrm{py}-H], 8.47\left[\mathrm{~d}, 2 \mathrm{H}, \mathrm{py}(-H)_{2}\right]$. IR $(\mathrm{KBr}): v_{\max } / \mathrm{cm}^{-1}=$ $3056 \mathrm{~m}(\mathrm{Ar}-\mathrm{H}), 2962 \mathrm{~m}(\mathrm{C}-\mathrm{H}), 2932 \mathrm{~m}(\mathrm{C}-\mathrm{H}), 2874 \mathrm{w}(\mathrm{C}-\mathrm{H})$, 1612 vs. $(\mathrm{C}=\mathrm{O}), 1448$ vs. (py), $1156 \mathrm{w}, 1064 \mathrm{~m}$ (py), 770 vs. (py), 750 m, $700 \mathrm{~m}$ (py), $594 \mathrm{~m}$. MS (ESI-TOF): $\mathrm{m} / z(\%)=414.117(100)$ $[\mathrm{M}-\mathrm{Cl}-\mathrm{Py}]^{+}, 412.114(80)[\mathrm{M}-\mathrm{Cl}-\mathrm{Py}]^{+}$.

$\left[\mathrm{Os}\left(\eta^{6}-p-c y m\right)(\mu-\mathrm{OH})(\mathrm{py})\right]_{2}\left(\mathrm{CF}_{3} \mathrm{SO}_{3}\right)_{\mathbf{2}}(\mathbf{1 6})$. It was obtained in an analogous manner to 13 but with $20 \mathrm{~h}$ reaction time, and using mebhaH. Recrystallisation of the crude product was made in ethylacetate. Yield: $67.58 \mathrm{mg}(0.072 \mathrm{mmol}, 36 \%)$. The obtained yellow crystals of $\mathbf{1 6}$ were suitable for X-ray structural analysis. Calcd. for $\mathrm{C}_{32} \mathrm{H}_{40} \mathrm{~F}_{6} \mathrm{~N}_{2} \mathrm{O}_{8} \mathrm{Os}_{2} \mathrm{~S}_{2}: \mathrm{C}, 33.74 ; \mathrm{H}, 3.54 ; \mathrm{N}$, 2.46; S, 5.63 found C, 33.68; H, 3.22; N, 2.39; S, 5.24. ${ }^{1} \mathrm{H}-\mathrm{NMR}$ (360 MHz, $d_{6}$-DMSO, $\left.298 \mathrm{~K}, \mathrm{TMS}\right): \delta=1.00[\mathbf{A}, \mathrm{d}, 12 \mathrm{H}$, $\left.\left(\left(-\mathrm{CH}_{3}\right)_{2}\right)_{2}\right], 1.02\left[\mathbf{B}, \mathrm{d}, 12 \mathrm{H},\left(\left(-\mathrm{CH}_{3}\right)_{2}\right)_{2}\right], 1.19[\mathbf{C}, \mathrm{d}, 12 \mathrm{H}$, $\left.\left(\left(-\mathrm{CH}_{3}\right)_{2}\right)_{2}\right], 1.76\left[\mathbf{B}, \mathrm{s}, 6 \mathrm{H},\left(-\mathrm{CH}_{3}\right)\right], 2.14\left[\mathbf{C}, \mathrm{s}, 6 \mathrm{H},\left(-\mathrm{CH}_{3}\right)\right], 2.25$ $\left[\mathrm{h}, 2 \mathrm{H}\left(-\mathrm{CH}\left(\mathrm{CH}_{3}\right)\right)_{2}\right], 2.37\left[\mathbf{A}, \mathrm{s}, 6 \mathrm{H},\left(-\mathrm{CH}_{3}\right)\right], 4.39[\mathbf{B}, \mathrm{s}, 2 \mathrm{H}$, $\mathrm{OH}], 5.03[\mathbf{C}, \mathrm{s}, 2 \mathrm{H}, \mathrm{OH}], 5.06[\mathbf{B}, \mathrm{d}, 4 \mathrm{H}, \mathrm{Ar}-H], 5.59[\mathbf{B}, \mathrm{d}, 4 \mathrm{H}$, $\mathrm{Ar}-H], 5.63[\mathbf{C}, \mathrm{d}, 4 \mathrm{H}, \mathrm{Ar}-H], 5.79[\mathbf{A}, \mathrm{d}, 4 \mathrm{H}, \mathrm{Ar}-H], 5.84[\mathbf{C}, \mathrm{d}$, $4 \mathrm{H}, \mathrm{Ar}-H], 5.92[\mathbf{A}, \mathrm{d}, 4 \mathrm{H}, \mathrm{Ar}-H], 6.90[\mathbf{A}, \mathrm{s}, 1 \mathrm{H}, \mathrm{OH}], 7.10[\mathbf{A}, \mathrm{t}$, $2 \mathrm{H}, \mathrm{Py}-H], 7.59[\mathbf{A}, \mathrm{t}, 1 \mathrm{H}, \mathrm{Py}-H], 7.82[\mathbf{B}, \mathrm{t}, 4 \mathrm{H}, \mathrm{Py}-H], 8.02[\mathbf{A}$, d, $2 \mathrm{H}, \mathrm{Py}-H], 8.18$ [B, t, 2H, Py-H], 8.79 [B, d, 4H, Py-H]. IR $(\mathrm{KBr}): v_{\max } / \mathrm{cm}^{-1}=3080 \mathrm{w}(\mathrm{Ar}-\mathrm{H}), 3052 \mathrm{w}(\mathrm{Ar}-\mathrm{H}), 2970 \mathrm{w}$ (C-H), $2928 \mathrm{w}(\mathrm{C}-\mathrm{H}), 2872 \mathrm{w}(\mathrm{C}-\mathrm{H}), 1450 \mathrm{~m}$ (py), 1282 vs, 1248 vs, $1226 \mathrm{~s}$ (triflate), $1160 \mathrm{~s}, 1030 \mathrm{vs}$. (triflate), $802 \mathrm{~m}$ (py), $636 \mathrm{~s}, 516 \mathrm{~m}$ (triflate). MS (ESI-TOF): $m / z(\%)=743.1929(100)\left[\mathrm{Os}_{2}\left(\eta^{6}-\right.\right.$ $p$-cym $\left.)_{2}(\mu-\mathrm{OMe})_{3}\right]^{+}$

$\left[\left\{\mathrm{Os}\left(\eta^{6}-p\right.\right.\right.$-cym)(meaha) $\left.\}(\mu-\mathrm{O})\left\{\mathrm{OsO}(\text { meaha })_{2}\right\}\right] \mathrm{CF}_{3} \mathrm{SO}_{3}$ (17). In an attempt to obtain single crystals of $\mathbf{4}$ to its solution in acetone, four fold hexane was layered and it was kept at $-20{ }^{\circ} \mathrm{C}$ for 2 weeks. As no solid formed, the solution was left to evaporate to dryness at room temperature. The resulting yellow crystals of $\mathbf{1 7}$ were found to be directly suitable for X-ray analysis.

\subsection{Cell lines and cell culture}

The in vitro anti-cancer chemotherapeutic potential of test compounds was determined using two human-derived malignant ovarian cancer cell lines (A2780 and A2780cisR). Both cell lines were a kind gift from Dr Maria Morgan, Dept. of Molecular \& Cellular Therapeutics, Royal College of Surgeons, Ireland, Dublin. A2780cisR cells are a cisplatin resistant human ovarian cell line developed by chronic exposure of the parent cisplatin sensitive A2780 cell line to increasing concentrations of cisplatin. Furthermore, these cells are cross-resistant to melphalan, adriamycin and irradiation. This resistant phenotype was maintained by pulsing cells during every third passage with cisplatin $(1 \mu \mathrm{M})$. Both cell lines were maintained in RPMI-1640 media with Earle's balanced salt solution (EBSS) containing $1.5 \mathrm{~g} \mathrm{~L}^{-1}$ sodium bicarbonate, $2 \mathrm{mM}$ L-glutamine, $100 \mathrm{U} \mathrm{ml}^{-1}$ penicillin, $100 \mathrm{~g} \mathrm{~m} \mathrm{ml}^{-1}$ streptomycin and $10 \%(\mathrm{v} / \mathrm{v})$ foetal bovine serum (FBS). These two model cell lines were grown at $37{ }^{\circ} \mathrm{C}$ in a humidified atmosphere with $5 \% \mathrm{CO}_{2}$ and were in the exponential phase of growth at the time of inclusion in cytotoxicity assays.

\subsection{Assessment of cytotoxicity, using MTT assay}

Each of the two cell lines $(100 \mu \mathrm{l})$ were seeded at a density of $2.5 \times 10^{4}$ cells $\mathrm{cm}^{-3}$ into sterile 96 well flat-bottomed plates (Sarstedt) and grown in $5 \% \mathrm{CO}_{2}$ at $37^{\circ} \mathrm{C}$. Test compounds were dissolved in DMF and diluted with culture media. The maximum percentage of DMF present in all wells was $0.5 \%(\mathrm{v} / \mathrm{v})$. Solutions $(100 \mu \mathrm{l})$ of complexes $1,5-8,10$ and 14 were added to replicate wells in the concentration range of $10-200 \mu \mathrm{M}$ and incubated for $72 \mathrm{~h}$. A miniaturised viability assay using 3-(4,5-dimethylthiazol2-yl)-2,5-diphenyl tetrazolium bromide (MTT) was carried out according to the method described by Mosmann. ${ }^{29}$ The $\mathrm{IC}_{50}$ value, defined as the drug concentration causing a 50\% reduction in cellular proliferation, was calculated for each complex. Each 
assay was carried out using five replicates and repeated on at least three separate occasions. Proliferation was calculated as a percentage of solvent-treated control cells, and expressed as a percentage of control. The significance of any reduction in cellular viability was determined using one-way ANOVA (analysis of variance). A probability of 0.05 or less was deemed statistically significant.

\section{Results and discussion}

\subsection{Synthesis and spectroscopic characterization}

$\left[\mathrm{M}\left(\eta^{6}-p \text {-cym }\right)(\mathrm{ha})\right]_{2}\left(\mathrm{CF}_{3} \mathrm{SO}_{3}\right)_{2}$ (4), [M( $\eta^{6}-p$-cym $\left.)(\mathrm{ha}) \mathrm{Cl}\right]$ (6) or $\left[\mathrm{M}\left(\eta^{6}-p\right.\right.$-cym $\left.)(\mathrm{ha})(\mathrm{py})\right] X(\mathbf{1 0})(M=\mathrm{Os}, \mathrm{Ru}$; ha = monohydroxamate, py $=$ pyridine, $X=\mathrm{Cl}^{-}$or $\mathrm{CF}_{3} \mathrm{SO}_{3}{ }^{-}$) type complexes were prepared in moderate to good yields from $\left[\mathrm{M}\left(\eta^{6}-p \text {-cym }\right) \mathrm{Cl}_{2}\right]_{2}$ precursors or from the coordinative unsaturated 2-3, respectively, by reacting them with the corresponding hydroxamates (see Chart 1) and with pyridine for 10-15 at room temperature. The novel compounds were air stable crystalline solids, and were soluble in polar solvents such as dichloromethane, acetone, dimethylsulfoxide, methanol and also water.

${ }^{1} \mathrm{H}-\mathrm{NMR}$ spectra of the complexes showed the expected resonance signals; and a representative sample spectrum of $\mathbf{5}$ is presented in Fig. S1, ESI.† Complexation resulted in downfield shifts $(0.05-0.11 \mathrm{ppm})$ of the $p$-cymene ring protons in $\mathbf{4 - 5}$ but highfield shifts $(0.18-0.33 \mathrm{ppm})$ for 6-9 when the corresponding resonances with those in the $\left[\mathrm{M}\left(\eta^{6}-p-c y m\right) \mathrm{Cl}_{2}\right]_{2}$ precursors were compared. This difference can be explained by an electron donating effect of the negatively charged chloride ion in the coordination sphere beside the hydroxamate in 6-9. Similarly, as a result of coordination of the hydroxamate $(0.02-0.61 \mathrm{ppm})$ or pyridine $(0.10-0.40 \mathrm{ppm})$ to the half-sandwich metal center, downfield shifts of the ligand protons are detected relative to the uncomplexed ligands except for the N2 and N6 protons of the pyridine ring which show highfield shifts. Furthermore, $\left[\mathrm{M}\left(\eta^{6}-p\right.\right.$-cym $)($ ha $\left.)(\mathrm{py})\right] X$ type complexes with the same ligands in the coordination sphere of the metal ion (e.g. 10 vs. 15), show practically identical chemical shift values, regardless of the $X$ counter ion.

The NMR spectrum of $\mathbf{1 6}$ indicated that three different species were formed in $\mathrm{d}^{6}$-DMSO solution after sample preparation using the solid crystalline complex. Selected parts of the spectrum are shown in Fig. S2, ESI. $\dagger$ DOSY experiments proved that besides free pyridine (D, Fig. S2), two other pyridine-containing complexes were present in solution, with three sets of signals belonging to the p-cymene protons being identifiable. Furthermore, three new singlets were also present in the spectrum which most probably belong to the hydroxide groups of the complexes. Complexes with the $\mathrm{M}(\mu-\mathrm{OH}) \mathrm{M}$ or $\mathrm{M}(\mu-\mathrm{OH})_{2} \mathrm{M}$ motif showed signals attributed to bridging $\mathrm{OH}$ in the $3.0-4.7 \mathrm{ppm}$ range. $^{30-32}$ Therefore, the data above may suggest that after dissolution of 16, partial or complete dissociation of the coordinated pyridine ligands occurred and besides free pyridine, it is possible that intact $\left[\mathrm{Os}\left(\eta^{6}-p \text {-cym }\right)(\mu-\mathrm{OH})(\mathrm{py})\right]_{2}{ }^{2+},\left[\mathrm{Os}\left(\eta^{6}-\right.\right.$ $p$-cym $)(\mathrm{py})(\mu-\mathrm{OH})_{2} \mathrm{Os}\left(\eta^{6}-p\right.$-cym $\left.)\right]^{2+}$ and $\left[\mathrm{Os}\left(\eta^{6}-p \text {-cym }\right)(\mu-\mathrm{OH})\right]_{2}{ }^{2+}$ ions are also present.

IR spectra of the novel hydroxamate complexes exhibited a new sharp band in the range $1545-1645 \mathrm{~cm}^{-1}$ compared to those of $\left[\mathrm{M}\left(\eta^{6}-p \text {-cym }\right) \mathrm{Cl}_{2}\right]_{2}$ precursors which was assigned to the $v_{\mathrm{CO}}$ of the coordinating hydroxamates. Comparison of the corresponding $\mathrm{Ru}$ and Os complexes indicated that these stretches appeared at slightly smaller wavenumbers for the former metal ion. The presence of the half-sandwich M(II) core was revealed by the characteristic five sharp bands in the wavenumber range of $3100-2850 \mathrm{~cm}^{-1} .8$ The triflate counter ion was indicated by the characteristic stretches at 1274, 1260, 1226, 1030 and $518 \mathrm{~cm}^{-1}, 33$ while coordinated pyridine was identified at 1448, 1065, 806, 771 and $701 \mathrm{~cm}^{-1}$.

Electrospray ionization mass spectrometric (ESI-MS) analysis in the positive mode provided further proof for the identity of the complexes. As found previously, ${ }^{8}$ the ESI-MS conditions produced no difference in the spectra to that of the corresponding triflate or chloride containing complexes (e.g. 4 vs. 6 or 5 vs. $7)$, regarding the major peaks. The same was observed with the $\left[\mathrm{M}\left(\eta^{6}-p\right.\right.$-cym $\left.)(\mathrm{ha})(\mathrm{py})\right] X$ complexes, revealing that chloride ion and pyridine dissociate at the ESI-MS conditions applied. All the mass spectra displayed the correct isotopic pattern.

\subsection{X-ray crystallographic studies}

Previously we have shown that in the presence of weakly coordinating counter ion like triflate, the primary $\left(R_{\mathrm{N}}=\mathrm{H}\right)$ hydroxamate ligand, bha ${ }^{-}$(Chart 1 ) was capable of bridging two half-sandwich $\left[\operatorname{Ru}\left(\eta^{6}-p \text {-cym }\right)\right]^{2+}$ units with the occupation of all coordination sites. $^{8}$ Our recent results demonstrate that a secondary $\left(R_{\mathrm{N}}=\mathrm{CH}_{3}\right)$ hydroxamate, meaha ${ }^{-}$(Chart 1$)$, binds to the metal ion in an identical manner. The ORTEP diagram of $\left[\operatorname{Ru}\left(\eta^{6}-p \text {-cym }\right)(\mu \text {-meaha })\right]_{2}{ }^{2+}(5)$ shown in Fig. 1. with key bond distances and angles appearing in the caption of Fig. 1. Regarding the $\mathrm{Ru}-\mathrm{O}$ distances, the bridging $\mathrm{Ru}-\mathrm{O}$ bonds [2.113(5)-2.159(5) $\AA$ ] were significantly longer than those of the Ru-carbonyl O bonds [2.065(5) $\AA$, 2.075(5) $\mathrm{A}]$. Comparison of the corresponding data with those determined previously ${ }^{8}$ for $\left[\mathrm{Ru}\left(\eta^{6}-p \text {-cym }\right)(\mu \text {-bha })\right]_{2}{ }^{2+}$ indicated no significant differences in the geometry and distances in the two dinuclear structures to

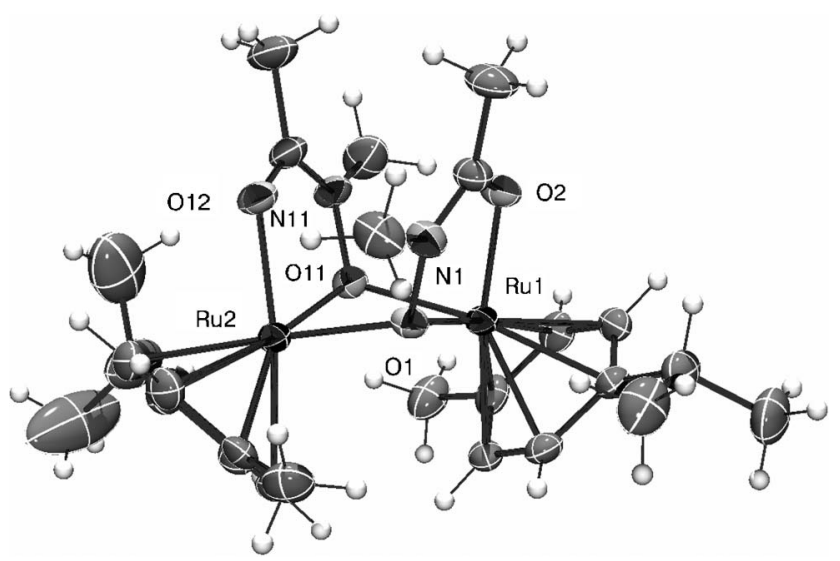

Fig. 1 X-Ray structure of the cation of $\left[\mathrm{Ru}\left(\eta^{6}-p\right.\right.$-cym)( $\mu$-meaha) $]_{2}\left(\mathrm{CF}_{3} \mathrm{SO}_{3}\right)_{2}(\mathbf{5})$. Thermal ellipsoids show $50 \%$ probability with partial numbering scheme. Selected bond lengths $(\AA)$ and angles $\left(^{\circ}\right)$ : $\mathrm{Ru}(1)-\mathrm{O}(1)$ 2.113(5), $\mathrm{Ru}(1)-\mathrm{O}(2)$ 2.065(5), Ru(1) $-\mathrm{O}(11)$ 2.151(5), $\mathrm{Ru}(2)$ $-\mathrm{O}(1)$ 2.160(5), $\mathrm{Ru}(2)-\mathrm{O}(12)$ 2.071(5), $\mathrm{Ru}(2)-\mathrm{O}(11)$ 2.112(5), $\mathrm{Ru}-$ $\mathrm{C}_{\text {arene }}($ avr.) 2.170(10); $\mathrm{O}(1)-\mathrm{Ru}(1)-\mathrm{O}(2)$ 76.8(2), O(1) $-\mathrm{Ru}(1)-\mathrm{O}(11)$ 77.9(2), $\mathrm{O}(2)-\mathrm{Ru}(1)-\mathrm{O}(11)$ 88.3(2), $\mathrm{O}(11)-\mathrm{Ru}(2)-\mathrm{O}(12)$ 76.3(2), O(11) $-\mathrm{Ru}(2)-\mathrm{O}(1)$ 77.7(2), O(12) - $\mathrm{Ru}(2)-\mathrm{O}(1)$ 87.3(2), Ru(1) -O(1) - Ru(2) 97.6(2), $\mathrm{Ru}(1)-\mathrm{O}(11)-\mathrm{Ru}(2) 97.9(2)$. 
that of the primary or secondary hydroxamate. However, with the appropriate Os precursors, we were able to obtain a pure crystalline solid only with secondary hydroxamates $\left(R_{\mathrm{N}}=\right.$ alkyl, aryl). This difference between the $\left[\mathrm{M}\left(\eta^{6}-p-c y m\right)\right]^{2+}(\mathrm{M}=\mathrm{Ru}, \mathrm{Os})$ cores can be explained by the $5 d$ osmium(II) being more easily involved in redox reactions. ${ }^{34}$ In particular, redox reactions with the oxidation of the metal ion by primary hydroxamates $\left(R_{\mathrm{N}}=\right.$ $\mathrm{H})$ yielding amide has been well documented in the literature for $\mathrm{Fe}(\mathrm{II})$ or $\mathrm{VO}(\mathrm{IV}) .^{35,36}$

The dinuclear complex formation was disfavoured in the presence of chloride ions which are capable of stronger interaction with the half-sandwich metal cores. As demonstrated with both metals, and with different hydroxamates, two of the coordination sites of the $\left[\mathrm{M}\left(\eta^{6}-p \text {-cym }\right)\right]^{2+}$ cores are taken by a hydroxamate $(\mathrm{O}, \mathrm{O})$ chelate, while chloride was present at the third position. A representative example of the ORTEP structure of $\left[\mathrm{Os}\left(\eta^{6}-p-c y m\right)(\right.$ meaha $\left.) \mathrm{Cl}\right]$ is shown is Fig. 2, while the structure of $\left[\mathrm{Ru}\left(\eta^{6}-p\right.\right.$-cym $)($ phebha $\left.) \mathrm{Cl}\right]$ in Fig. S3, ESI. $\uparrow$ The corresponding bond distances and angles (captions to Fig. 2 and S3) revealed no significant differences when the two metals or the different type hydroxamate ligands were compared. Both the Os- $\mathrm{Cl}(2.425 \AA)$ and the $\mathrm{Ru}-\mathrm{Cl}(2.411 \AA)$ distances were shown to be in the expected range (Os: $2.40(7), \mathrm{Ru}: 2.42(6) \AA) .{ }^{37}$

Unexpectedly, we have found the formation of an unusual dinuclear Os complex (17) with the partial oxidation of the metal ion, when a solution of $\left[\mathrm{Os}\left(\eta^{6}-p \text {-cym }\right)(\text { meaha })\right]_{2}\left(\mathrm{CF}_{3} \mathrm{SO}_{3}\right)_{2}(4)$ in a mixture of acetone/hexane, was left to evaporate slowly under aerobic conditions, and at room temperature in a narrow crystallization tube. The X-ray structure of the isolated yellow solid is presented in Fig. 3, while the key bond angles and distances are summarized in the caption to Fig. 3. It can be seen that the new dinuclear complex consisted of a half-sandwich [Os $\left(\eta^{6}-p\right.$-cym) $]$ core to which a meaha ${ }^{-}$ligand is bound via $(\mathrm{O}, \mathrm{O})$ chelate. This unit was linked via an oxo group to another

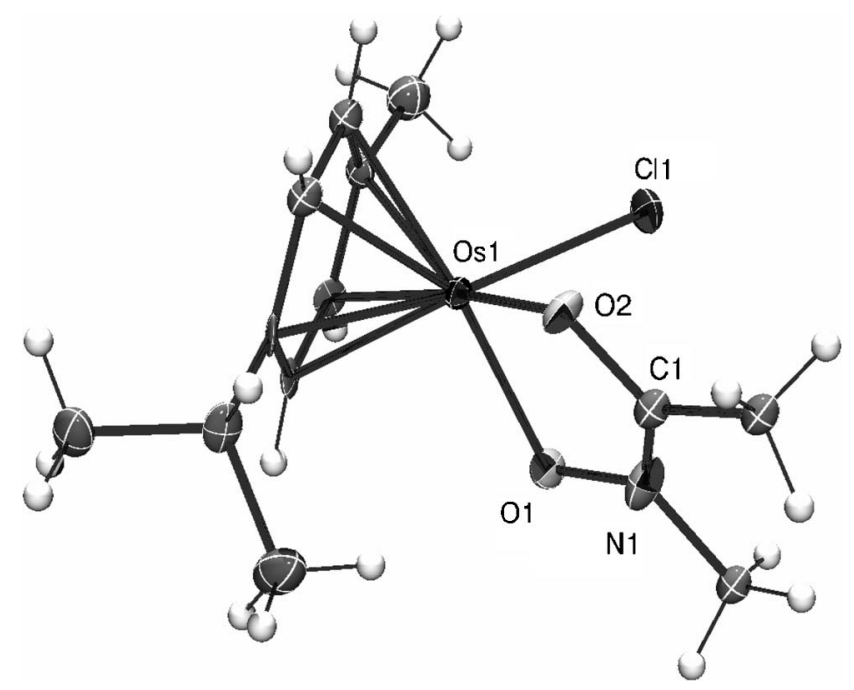

Fig. 2 X-Ray structure of $\left[\mathrm{Os}\left(\eta^{6}-p\right.\right.$-cym $)($ meaha $\left.) C l\right]$ (6). Thermal ellipsoids show $50 \%$ probability with partial numbering scheme. Selected bond lengths $(\AA)$ and angles ( $\left.{ }^{\circ}\right)$ : Os(1) $-\mathrm{Cl}(1) 2.429(2)$, Os(1) $\mathrm{O}(1) 2.075(4), \mathrm{Os}(1)-\mathrm{O}(2)$ 2.092(4), Os(1) $-\mathrm{C}_{\text {arene }}(\mathrm{avr}$.) 2.172(25); $\mathrm{O}(1)-$ $\mathrm{Os}(1)-\mathrm{O}(2)$ 76.68(15), $\mathrm{Cl}(1)-\mathrm{Os}(1)-\mathrm{O}(1)$ 82.19(11), $\mathrm{Cl}(1)-\mathrm{Os}(1)-\mathrm{O}(2)$ 82.99(11).

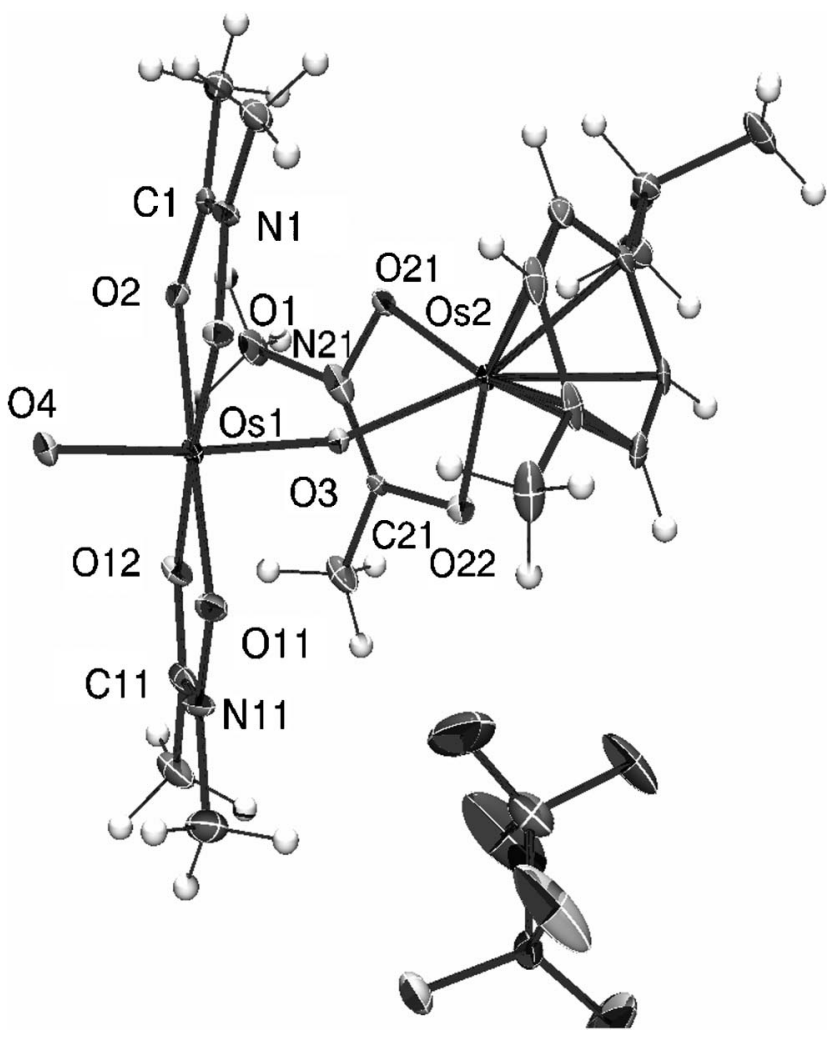

Fig. 3 X-Ray structure of $\left[\left\{\mathrm{Os}\left(\eta^{6}-p\right.\right.\right.$-cym $)($ meaha $\left.\left.)\right\}(\mu-\mathrm{O})\left\{\mathrm{OsO}(\text { meaha })_{2}\right\}\right]-$ $\mathrm{CF}_{3} \mathrm{SO}_{3}$ (17). Thermal ellipsoids show $50 \%$ probability with partial numbering scheme. Selected bond lengths $(\AA)$ and angles $\left({ }^{\circ}\right)$ : Os(1) $-\mathrm{O}(1)$ 1.979(2), Os(1) $-\mathrm{O}(2)$ 2.054(2), Os(1) $-\mathrm{O}(3)$ 1.781(2), Os(1) $-\mathrm{O}(4)$ 1.718(3), Os(1) $-\mathrm{O}(11)$ 1.977(3), Os(1) $-\mathrm{O}(12) 2.058(3), \mathrm{Os}(2)-\mathrm{O}(3)$ 2.035(2)Os(2) $\mathrm{O}(21)$ 2.063(3), Os(2) - O(22) 2.058(2), Os(1) - $\mathrm{C}_{\text {arene }}$ (avr.) 2.178(14); Os(1) $\mathrm{O}(3)-\mathrm{Os}(2)$ 162.58(14), $\mathrm{O}(21)-\mathrm{Os}(2)-\mathrm{O}(22) 78.07(10), \mathrm{O}(21)-\mathrm{Os}(2)-\mathrm{O}(3)$ 82.51(10), O(22) - Os(2) -O(3) 81.74(10), O(3) - Os(1)-O(4) 169.84(13).

metal core in which an osmium with octahedral geometry was located. The remaining coordination sites of the second Os were occupied by two $(\mathrm{O}, \mathrm{O})$ chelates of two meaha ${ }^{-}$ligands and an oxo group. Comparison of the $\mathrm{Os}(2)-\mathrm{O}(3)(2.035 \AA), \mathrm{Os}(1)-\mathrm{O}(3)$ $(1.781 \AA)$ distances and the $\mathrm{Os}(1)-\mathrm{O}(3)-\mathrm{Os}(2)$ bond angle $\left(162^{\circ}\right)$ with those of dinuclear oxo bridged Os complexes ${ }^{38,39}$ also supports the theory that an oxo group, and not a hydroxide ion, binds the two metal cores together. While octahedral Os complexes with an Os-O single bond feature 2.09(6) $\AA$ while with an Os=O double bond 1.73(3) $\AA$ distances ${ }^{37}$ the short value of Os(1)-(O4) $(1.718 \AA$ ) in $\mathbf{1 7}$ is indicative for an $\mathrm{Os}=\mathrm{O}$ bond. As the complex also contains a non-coordinating chloride ion the second osmium should have a +6 oxidation state in 17. Similar oxocationic octahedral osmium complexes with the $\mathrm{OsO}^{4+}$ core have already been reported in the literature with $(\mathrm{O}, \mathrm{O})$ ligands. ${ }^{40-42}$

Dinuclear complex formation can also be hindered if other monodentate ligands capable of relatively strong coordination to the half-sandwich metal cores (e.g. pyridine) are present beside the coordinating hydroxamate. As an example, the crystal structure of $\left[\mathrm{Ru}\left(\eta^{6}-p\right.\right.$-cym $\left.)(\mathrm{bha})(\mathrm{py})\right] \mathrm{CF}_{3} \mathrm{SO}_{3}$ (12) appears in Fig. 4, while that of $\left[\mathrm{Ru}\left(\eta^{6}-p\right.\right.$-cym $)($ phebha $\left.)(\mathrm{py})\right] \mathrm{CF}_{3} \mathrm{SO}_{3}(\mathbf{1 4})$ is shown in Fig. S4, ESI. $\dagger$ In both structures, beside the hydroxamate $(\mathrm{O}, \mathrm{O})$ chelate, a pyridine $\mathrm{N}$ can be found in the third coordination site of the metal ion. 14 is also stabilized by 


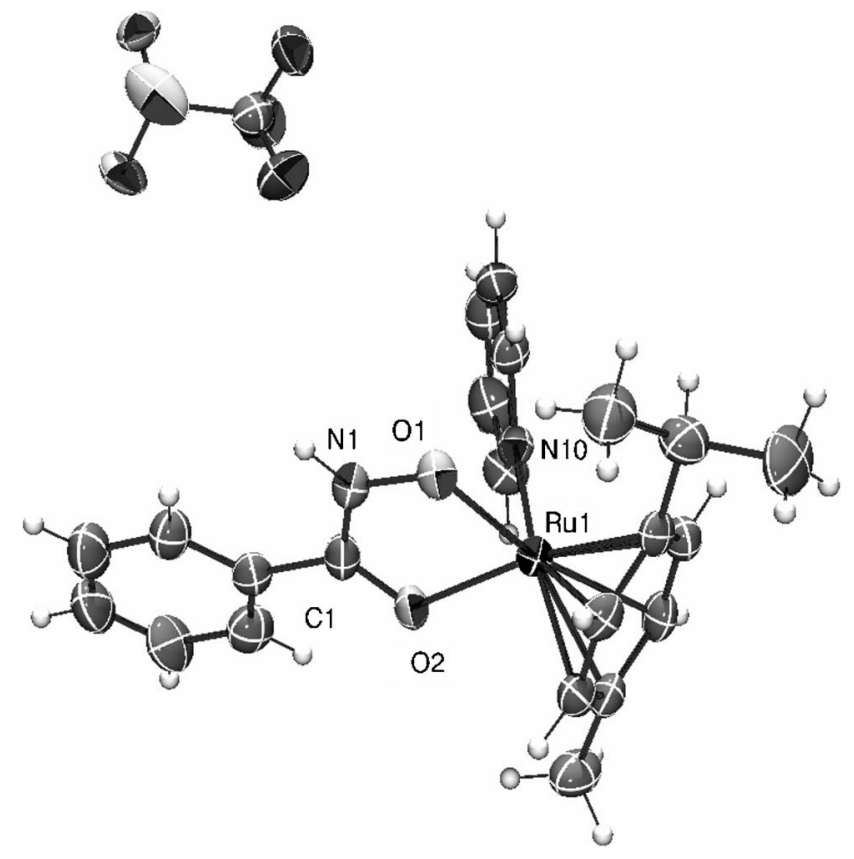

Fig. 4 X-Ray structure of $\left[\mathrm{Ru}\left(\eta^{6}-p\right.\right.$-cym)(bha)(py) $] \mathrm{CF}_{3} \mathrm{SO}_{3} \quad$ (12). Thermal ellipsoids show $50 \%$ probability with partial numbering scheme. Selected bond lengths $(\AA)$ and angles $\left(^{\circ}\right)$ : $\mathrm{Ru}(1)-\mathrm{O}(1)$ 2.044(7), $\mathrm{Ru}(1)-$ $\mathrm{O}(2)$ 2.084(6), $\mathrm{Ru}(1)-\mathrm{N}(10)$ 2.123(8), $\mathrm{Ru}(1)-\mathrm{C}_{\text {arene }}$ (avr.) 2.169(23); $\mathrm{O}(1)$ $-\mathrm{Ru}(1)-\mathrm{O}(2)$ 78.3(3), N(10) - $\mathrm{Ru}(1)-\mathrm{O}(1)$ 83.1(3), N(10) - $\mathrm{Ru}(1)-\mathrm{O}(2)$ 82.1(3).

hydrogen bonds which can be detected between the hydroxamate NH's as donors and triflate O's as acceptors. The dimensions of these $\mathrm{H}$ bonds are as follows: $\mathrm{N}(1) \ldots \mathrm{O}(81)=2.799(13) \AA$, $\mathrm{H}(\mathrm{N} 1) \ldots \mathrm{O}(81)=1.98(4) \AA, \mathrm{N}(1)-\mathrm{H}(\mathrm{N} 1) \ldots \mathrm{O}(81)=160(10)^{\circ}$ and $\mathrm{N}(11) \ldots \mathrm{O}(73 \mathrm{i})=2.891(12) \AA, \mathrm{H}(\mathrm{N} 11) \ldots \mathrm{O}(73 \mathrm{i})=2.13(6) \AA$, $\mathrm{N}(11)-\mathrm{H}(\mathrm{N} 11) \ldots \mathrm{O}(73 \mathrm{i})=148(9)^{\circ}$. Comparison of the corresponding bond length and angle values (captions to Fig. 4 and S4) indicates that benzohydroxamate $\left(R_{\mathrm{C}}=\right.$ Phe, $\left.R_{\mathrm{N}}=\mathrm{H}\right)$ and N-phenyl-benzohydroxamate $\left(R_{\mathrm{C}}=R_{\mathrm{N}}=\right.$ Phe $)$, the latter with larger steric demand, may behave very similarly in these mixed complexes.

Although we do not have an X-ray structure of $\mathbf{1 5}$ with the meaha $^{-}$ligand, the obtained analytical data are consistent with its stoichiometry. At the same time, during the synthesis of the analogous Os complex with mebhaH, we were unsuccessful in obtaining $\left[\mathrm{Os}\left(\eta^{6}-p\right.\right.$-cym $)($ mebha $\left.)(\mathrm{py})\right] \mathrm{CF}_{3} \mathrm{SO}_{3}$. Instead, using $20 \mathrm{~h}$ reaction time and after work-up of the reaction mixture, a novel dihydroxo bridged mixed pyridine complex, 16, could be isolated and the X-ray structure determined. As Fig. 5 reveals, this symmetrical dinuclear Os complex has two half-sandwich $\left[\mathrm{Os}\left(\eta^{6}-p \text {-cym }\right)(\mathrm{py})\right]^{+}$units which are linked via hydroxide bridges. Key bond lengths and angles are summarized in the caption to Fig. 5. Os-OH distances (2.088(9) and 2.091(9) $\AA$ ) in $\mathbf{1 6}$ are in the range similar to that of Os complexes, showing an average Os- $\mathrm{OH}$ distance ${ }^{37}$ of 2.11(5) $\AA$ for the published four structures having hydroxo bridges between the two osmium atoms. The appropriate data for $\mathbf{1 6}$ is also in good agreement with the published Os-O(H)-Os angles (average: $103^{\circ}$ ) and Os-Os distances, average of 3.23(7) $\AA$. Formation of 16 is likely due to the decomposition of the mebha ${ }^{-}$ligand, and to the

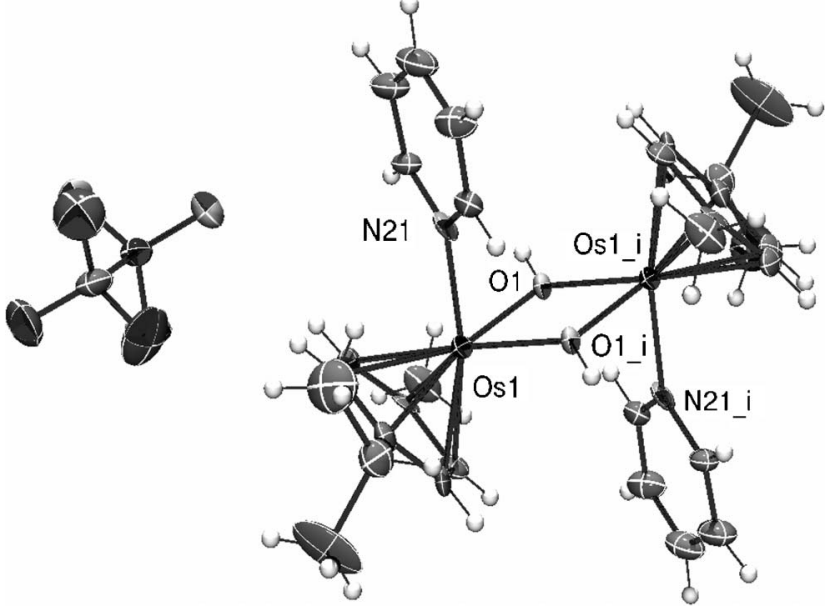

Fig. 5 X-Ray structure of $\left[\mathrm{Os}\left(\eta^{6}-p \text {-cym }\right)(\mu-\mathrm{OH})(\mathrm{py})\right]_{2}\left(\mathrm{CF}_{3} \mathrm{SO}_{3}\right)_{2}$ (16). Thermal ellipsoids show $50 \%$ probability with partial numbering scheme. Selected bond lengths $(\AA)$ and angles $\left({ }^{\circ}\right)$ : Os(1) -O(1) 2.091(9), Os(1) $\mathrm{O}\left(1 \_\right.$i) 2.088(9), Os(1_i) -O(1) 2.088(9), Os(1) - N(21) 2.104(11), Os(1) $\mathrm{C}_{\text {arene }}$ (avr.) 2.182(20); Os(1)-O(1)-Os(1_i) 107.3(4), O(1) -Os(1) - N(21) 84.6(4), O(1) -Os(1) - O(1_i) 72.7(4), O(1_i) -Os(1) - N(21) 81.5(4).

formation of kinetically inert hydroxo bridged species, with the involvement of trace water present during the reaction or subsequent work-up.

\subsection{Cytotoxicity in cancer cell lines}

The in vitro anti-cancer chemotherapeutic potential of this series of Ru- and Os-hydroxamic acid complexes was determined using two human-derived ovarian cancer cell lines; a parental cell line (A2780) and a cisplatin-resistant variant (A2780 cisR). The results show that all the complexes (1) can be regarded as inactive $\left(\mathrm{IC}_{50}>200 \mu \mathrm{M}\right)$, since they failed to reduce the viability of either model cell line across both the concentration range $(0-$ $200 \mu \mathrm{M})$ and incubation period $(72 \mathrm{~h})$ studied. In contrast, cisplatin displayed a significant reduction in cellular viability toward both cell lines, with mean $\mathrm{IC}_{50}$ values of $1.3 \pm 0.1$ and $9.7 \pm 1.0 \mu \mathrm{M}$ seen in A2780 and A2780 cisR cells, respectively.

\section{Conclusions}

The results of this paper showed that secondary monohydroxamates $\left(R_{\mathrm{N}}=\right.$ alkyl, aryl $)$ were capable of the double bridging of two half-sandwich M(II) ( $M=\mathrm{Ru}$, Os) cores, and the same holds true for the primary hydroxamate $\left(R_{\mathrm{N}}=\mathrm{H}\right)$ complexes of $\mathrm{Ru}$. The X-ray crystal structure of $\mathbf{5}$ demonstrated that in the dinuclear entity, the two meaha ${ }^{-}$ligands were bound in an identical manner via deprotonated O's as bridging atoms and carbonyl O's as monodentate atoms. As $\left[\mathrm{Os}\left(\eta^{6}-p-c y m\right)\right]^{2+}$ was less resistant to redox reactions, it was not possible to isolate stable complexes with primary hydroxamates. The presence of monodentate co-ligands resulted in the formation of mononuclear complexes with the expected $\left[\mathrm{M}\left(\eta^{6}-p \text {-cym }\right)(\text { ha }) \mathrm{X}\right]^{\mathrm{n}+}(M$ = Os, $\mathrm{Ru}$; ha = hydroxamate, $X=\mathrm{py}, \mathrm{Cl}^{-}$) stoichiometry, with [Os $\left(\eta^{6}-p\right.$-cym $)($ meaha $\left.) \mathrm{Cl}\right](6)$, being the first organometallic Os(II)-hydroxamate characterized by X-ray crystallography. The unexpected formation of an oxo bridged dinuclear $\mathrm{Os}^{\mathrm{VI}}$ / Os ${ }^{\mathrm{II}}$ complex, 17, consisting an octahedral Os(VI) core and an 
intact half-sandwich $\left[\mathrm{Os}\left(\eta^{6}-p-\mathrm{cym}\right)\right]^{2+}$ unit suggests that partial oxidation of the $\left[\mathrm{Os}\left(\eta^{6}-p \text {-cym }\right)\right]^{2+}$ core may happen under aerobic conditions.

Complexes 1, 5-8, 10 and $\mathbf{1 4}$ were screened for possible in vitro anti-proliferative activity. Results showed that $\mathrm{IC}_{50}$ values were greater than $200 \mu \mathrm{M}$. This may be explained by fast ligand exchange reactions of the $(\mathrm{O}, \mathrm{O})$ donor hydroxamates. In the case of the Os complexes, it is possible that dissociation of administered compounds, may lead to the formation of less active species which are incapable of biological activity at low concentrations. However, given that similar complexes have previously shown antimicrobial activity, additional biological studies will focus on an assessment of their anti-bacterial (Gram positive and Gram negative) and anti-fungal profile.

\section{Acknowledgements}

We thank members of the EU COST Action D39 for motivating discussions. This work was supported by the Hungarian Scientific Research Fund (OTKA K76142), TAMOP 4.2.1./B09/1/KONV-2010-0007 project co-financed by the European Union and the European Social Fund by the Technological Sector Research Programme, Strand III, under the European Social Fund and the Programme for Research in Third Level Institutes (2006-2010).

\section{References}

1 C. J. Marmion, D. Griffith and K. B. Nolan, Eur. J. Inorg. Chem., 2004, 3003

2 P. A. Marks, Oncogene, 2007, 26, 1351.

3 P. A. Marks and W. S. Xu, J. Cell. Biochem., 2009, 107, 600.

4 M. Melchart, P. J. Sadler, in: Bioorganometallics, (ed. G. Jaouen), Wiley-VCH, 2006, p. 39-64.

5 P. C. A. Bruijnincx and P. J. Sadler, Adv. Inorg. Chem., 2009, 61, 1.

6 I. Bratsos, T. Gianferrara, E. Alessio, C. G. Hartinger, M. A. Jakupec and B. K. Keppler, in: Bioinorganic Medicinal Chemistry, ed. E. Alessio, Wiley-VCH, 2011, p. 160-164.

7 I. Bratsos, D. Urankar, E. Zangrando, P. Genova-Kalou, J. Kosmrlj, E. Alessio and I. Turel, Dalton Trans., 2011, 40, 5188.

8 P. Buglyó and E. Farkas, Dalton Trans., 2009, 8063.

9 L. Bíró, E. Farkas and P. Buglyó, Dalton Trans., 2010, 39, 10272.

10 P. Buglyó and D. Griffith, unpublished results.

11 A. F. A. Peacock, A. Habtemariam, R. Fernández, V. Walland, F. P. A. Fabbiani, S. Parsons, R. E. Aird, D. I. Jodrell and P. J. Sadler,

J. Am. Chem. Soc., 2006, 128, 1739.

12 A. F. A. Peacock, A. Habtemariam, S. A. Moggach, A. Prescimone, S. Parsons and P. J. Sadler, Inorg. Chem., 2007, 46, 4049.
13 L. K. Filak, G. Mühlgassner, M. A. Jakupec, P. Heffeter, W. Berger, V. B. Arion and B. K. Keppler, JBIC, J. Biol. Inorg. Chem., 2010, 15, 903.

14 L. K. Filak, G. Mühlgassner, F. Bacher, A. Roller, M. Galanski, M. A. Jakupec, B. K. Keppler and V. B. Arion, Organometallics, 2011, 30, 273 .

15 A. F. A. Peacock, S. Parsons and P. J. Sadler, J. Am. Chem. Soc., 2007, 129, 3348

16 R. Schuecker, R. O. John, M. A. Jakupec, V. B. Arion and B. K. Keppler, Organometallics, 2008, 27, 6587.

17 A. F. A. Peacock, M. Melchart, R. J. Deeth, A. Habtemariam, S. Parsons and P. J. Sadler, Chem.-Eur. J., 2007, 13, 2601.

18 A. Das, F. Basuli, L. R. Falvello and S. Bhattacharya, Inorg. Chem., 2001, 40, 4085.

19 D. D. Perrin and W. L. F. Armarego, Purification of Laboratory Chemicals, 3rd Edition, Pergamon, Oxford, 1988.

20 D. A. Brown, R. A. Geraty, J. D. Glennon and N. N. Choileain, Synth. Commun., 1985, 15, 1159.

21 B. Monzyk and A. L. Crumbliss, J. Org. Chem., 1980, 45, 4670.

22 M. A. Bennett, T. W. Matheson, G. B. Robertson, A. K. Smith and P. A. Tucker, Inorg. Chem., 1980, 19, 1014.

23 M. A. Bennett and A. K. Smith, J. Chem. Soc., Dalton Trans., 1974, 233.

24 A. Schlüter, K. Bieber and W. S. Sheldrick, Inorg. Chim. Acta, 2002, 340, 35.

25 W. A. Kiel, R. G. Ball and W. A. G. Graham, J. Organomet. Chem., 1990, 383, 481.

26 A. Altomare, G. Cascarano, C. Giacovazzo and A. Guagliardi, J. Appl. Crystallogr., 1993, 26, 343.

27 G. M. Sheldrick, Acta Crystallogr., Sect. A: Found. Crystallogr., 2008, A64, 112

28 L. J. Farrugia, J. Appl. Crystallogr., 1999, 32, 837.

29 T. Mosmann, J. Immunol., 1983, 65, 55.

30 D. Carmona, A. Mendoza, J. Ferrer, F. J. Lahoz and L. A. Oro, J. Organomet. Chem., 1992, 431, 87.

31 Q. F. Zang, R. D. Adams and W. H. Leung, Inorg. Chim. Acta, 2006, 359, 978

32 M. Auzias, B. Therrien and G. Süss-Fink, Inorg. Chem. Commun., 2007, 10, 1239.

33 J. M. Alía, Y. Díaz de Mera, H. G. M. Edwards, F. J. García and E. E. Lawson, J. Mol. Struct., 1997, 408(409), 439-450.

34 F. A. Cotton and G. Wilkinson, Advanced Inorganic Chemistry, 5th Edition, Wiley, New York, 1988.

35 E. Farkas, É. A. Enyedy and I. Pócsi, J. Inorg. Biochem., 2004, 98, 1957.

36 P. Buglyó and N. Pótári, Polyhedron, 2005, 24, 837.

37 Cambridge Structural Database, Version 5.32 Update May, 2011.

38 J. A. Christie, T. J. Collins, T. E. Krafft, B. D. Santarsiero and G. H. Spies, Chem. Commun., 1984, 198.

39 C. L. Gross, J. L. Brumaghim and G. S. Girolami, Organometallics, 2007, 26, 2258.

40 T. Behling, M. V. Capparelli, A. C. Skapski and G. Wilkinson, Polyhedron, 1982, 1, 840 .

41 A. Struess and W. Preetz, Z. Naturforsch. B, 1998, 53, 823.

42 S. Stanislas, A. L. Beauchamp and C. Reber, Inorg. Chem., 2000, 39, 2152. 